\title{
GLOBAL ASYMPTOTIC BEHAVIOR OF ITERATIVE IMPLICIT SCHEMES*
}

\author{
H. C. YEE \\ NASA Ames Research Center, Moffett Field, CA 94035, USA \\ P. K. SWEBY \\ University of Reading, Whiteknights, Reading RG6 2AX, England \\ Received January 14, 1994; Revised March 16, 1994
}

\begin{abstract}
The global asymptotic nonlinear behavior of some standard iterative procedures in solving nonlinear systems of algebraic equations arising from four implicit linear multistep methods (LMMs) in discretizing three models of $2 \times 2$ systems of first-order autonomous nonlinear ordinary differential equations (ODEs) is analyzed using the theory of dynamical systems. The iterative procedures include simple iteration and full and modified Newton iterations. The results are compared with standard Runge-Kutta explicit methods, a noniterative implicit procedure, and the Newton method of solving the steady part of the ODEs. Studies showed that aside from exhibiting spurious asymptotes, all of the four implicit LMMs can change the type and stability of the steady states of the differential equations (DEs). They also exhibit a drastic distortion but less shrinkage of the basin of attraction of the true solution than standard nonLMM explicit methods. The simple iteration procedure exhibits behavior which is similar to standard nonLMM explicit methods except that spurious steady-state numerical solutions cannot occur. The numerical basins of attraction of the noniterative implicit procedure mimic more closely the basins of attraction of the DEs and are more efficient than the three iterative implicit procedures for the four implicit LMMs. Contrary to popular belief, the initial data using the Newton method of solving the steady part of the DEs may not have to be close to the exact steady state for convergence. These results can be used as an explanation for possible causes and cures of slow convergence and nonconvergence of steady-state numerical solutions when using an implicit LMM time-dependent approach in computational fluid dynamics.
\end{abstract}

\section{Background and Objective}

It has been shown recently by the authors and others [Yee et al., 1991; Yee \& Sweby, 1992; Lafon \& Yee, 1991; Lafon \& Yee, 1992; Griffiths et al., 1992a,b; Sweby \& Yee, 1991; Yee et al., 1992; Mitchell \& Griffiths, 1985; Iserles, 1988; Iserles et al., 1990; Stuart, 1989; Dieci \& Estep,
1990] that the dynamics of the numerical discretizations of nonlinear differential equations (DEs) can differ significantly from that of the original DEs themselves. For example, it was shown in Yee et al. [1991], Yee \& Sweby [1992], and Lafon \& Yee $[1991,1992]$ that the discretizations can possess spurious steady-state numerical solutions and

\footnotetext{
*Written version of the paper presented at the SIAM Conference on Applications of Dynamical Systems, October 15-19, 1992, Salt Lake City, Utah; a condensed version will appear in the Proceedings of the Chaotic Numerics Workshop, July 12-16, 1993, Deakin University, Geelong, Australia.
} 
spurious asymptotes which do not satisfy the original DEs. These spurious numerical solutions may be stable or unstable and may occur both below and above the linearized stability limit of the numerical scheme (on the time step for the equilibrium or asymptote of the DE). In Yee \& Sweby [1992], Lafon \& Yee [1991], Sweby \& Yee [1991], and Yee et al. [1992a] we showed how "numerical" basins of attraction can complement the bifurcation diagrams in obtaining the global asymptotic behavior of numerical solutions for nonlinear DEs. We showed how in the presence of spurious asymptotes the basins of the true stable steady states could be altered by the basins of the spurious stable and unstable asymptotes. One major consequence of this phenomenon which is not commonly known is that this spurious behavior can result in a dramatic distortion and, in most cases, a dramatic shrinkage and segmentation of the basin of attraction of the true solution for finite time steps. Such distortion, shrinkage, and segmentation of the numerical basins of attraction will occur regardless of the stability of the spurious asymptotes.

We use the term "spurious asymptotic numerical solutions" to mean asymptotic solutions that satisfy the discretized counterparts but do not satisfy the underlying ordinary differential equations (ODEs) or partial differential equations (PDEs). In other words, asymptotic solutions that are asymptotes of the discretized counterparts but are not asymptotes of the DEs. Asymptotic solutions here include steady-state solutions, periodic solutions, limit cycles, chaos, and strange attractors. Here, the basin of attraction is a domain of a set of initial conditions whose solution curves (trajectories) all approach the same asymptotic state. Also, we use the term "exact" and "numerical" basins of attraction to distinguish "basins of attraction of the underlying DEs" and "basins of attraction of the discretized counterparts".

In view of the above nonlinear behavior of numerical schemes, it is possible that numerical computations may converge to an incorrect steady state or other asymptotes which appear to be physically reasonable. One major implication is that what is expected to be physical initial data associated with a true steady state might lead to a wrong steady state, a spurious asymptote, or a divergence or nonconvergence of the numerical solution. In addition, the existence of spurious limit cycles [Yee et al., 1992; Yee et al., 1991; Yee \& Sweby, 1992] may result in the type of nonconvergence of steady-state numerical solutions observed in time-dependent approaches to the steady states. It is our belief that the understanding of the symbiotic relationship between the strong dependence on initial data and permissibility of spurious stable and unstable asymptotic numerical solutions at the fundamental level can guide the tuning of the numerical parameters and the proper and/or efficient usage of numerical algorithms in a more systematic fashion. It can also explain why certain schemes behave nonlinearly in one way but not another. Here, strong dependence on initial data means that for a finite time step $\Delta t$ that is not sufficiently small, the asymptotic numerical solutions and the associated numerical basins of attraction depend continuously on the initial data. Unlike nonlinear problems, the associated numerical basins of attraction of linear problems are independent of $\Delta t$ as long as $\Delta t$ is below a certain upper bound.

Studies in Yee et al. [1991], Yee \& Sweby [1992], Lafon \& Yee [1991, 1992], Griffiths et al. [1992a,b], Sweby \& Yee [1991], Yee et al. [1992], Mitchell \& Griffiths [1985], Iserles [1988], Iserles et al. [1990], Stuart [1989], and Dieci \& Estep [1990] are particularly important for computational fluid dynamics (CFD), since it is a common practice in CFD computations to use a time-dependent approach to obtain steady-state numerical solutions of complicated steady fluid flows which often consist of stiff nonlinear PDEs of the mixed type. When a timedependent approach is used to obtain steady-state numerical solutions of a fluid flow or a steady PDE, a boundary value problem is transformed into an initial-boundary value problem with unknown initial data. If the steady PDE is strongly nonlinear and/or contains stiff nonlinear source terms, phenomena such as slow convergence, nonconvergence or spurious steady-state numerical solutions commonly occur even though the time step is well below the linearized stability limit and the initial data are physically relevant.

It is also a common practice in CFD to use implicit methods to solve stiff problems. Such schemes, however, introduce the added difficulty of solving the implicit equations (nonlinear algebraic equations) in order to obtain the solution at the next time level. Various options such as linearization or iteration are available for this purpose. In Yee \& Sweby [1992], we included a study on the dynamics of a noniterative linearized version of the implicit Euler and trapezoidal methods. In this work we generalize our earlier work [Yee \& Sweby, 1992] to 
include iterative solution procedures of the implicit discretized equations, namely, simple iteration and full and modified Newton iteration. In addition, we also study the 3-level backward differentiation formula (BDF) and a midpoint implicit method (onelegged method of the trapezoidal method) with these four methods (noniterative versus iterative) of solving the resulting nonlinear algebraic equations applied to it. A comparison of the above combination of implicit methods with the nine explicit methods studied in Yee \& Sweby [1992], and with the "straight" Newton method in solving the steady part of the equations, is also performed. We use the term "straight Newton" to distinguish it from the combination of "implicit LMM + Newton" type of solution procedure. Some study on variable time step control to avoid spurious asymptotes will also be addressed. A condensed version of this work will appear in Yee \& Sweby [1993]. Generalization of the study to include grid adaptation as one of the sources of nonlinearity and/or stiffness that is introduced into the discretized system will be reported in Sweby \& Yee [1994] and Budd et al. [1994].

\section{Relevance and Outline}

Since all four implicit methods under consideration are linear multistep methods (LMMs) they will not exhibit spurious steady states (fixed points of order one). However, as discussed in Yee \& Sweby [1992] and as we shall see in later sections, some implicit LMMs have the property of increasing the stability range for the stable fixed points of the ODE [Dieci \& Estep, 1990], accompanied in some instances by the stabilization of unstable fixed points of the ODE [Yee \& Sweby, 1992]. In addition, the method of solution of the implicit equations generated by these schemes can itself contribute to the dynamics of the discretization since different numerical methods and/or solution procedures result in entirely different nonlinear discrete maps. Iserles [1988] and Dieci \& Estep [1990] were the first to examine some of the stability issues. Our attempt here is to address issues that were not investigated in Iserles [1988] and Dieci \& Estep [1990], and in our companion paper [Yee \& Sweby, 1992]. Our main purpose is to study the global asymptotic behavior in terms of bifurcation diagrams and numerical basins of attraction of these four procedures for solving nonlinear systems of algebraic equations arising from implicit LMM discretizations.
Although we purposely selected the model equations with known analytical solutions, depending on the scheme, the dynamics of their discretized counterpart are very difficult and might not be possible to analyze analytically. Only some analysis is possible. Part of the global asymptotic numerical solution behavior can be obtained by the pseudo arclength continuation method devised by Keller [1977], a standard numerical method for obtaining bifurcation curves in bifurcation analysis. Besides not being able to provide the numerical basins of attraction, one deficiency of the pseudo arclength continuation method is that, for problems with complicated bifurcation patterns, it cannot provide the complete bifurcation diagram without a known solution for each of the main bifurcation branches. For spurious asymptotes it is usually not easy to locate even just one solution on each of these branches. For the majority of the cases where rigorous analysis is impractical, we utilized numerical experiments. Also, analytical representations (except in isolated cases) for numerical basins of attraction rarely exist for nonlinear DEs. Methods such as generalized cell mapping [Hsu et al., 1982; Flashner \& Guttalu, 1988] can provide an efficient approach to locating these basins, but might not be exact. Here, our aim is to numerically compute the basins of attraction as accurately as possible and in the most straightforward way in order to illustrate the key points.

Due to the complicated nature of these discrete maps, analysis without a supercomputer is nearly impossible. The nature of our studies requires the performance of a very large number of simulations with different initial data; this can be achieved by use of the highly parallel Connection Machines (CM-2 or CM-5) whereby each processor could represent a single initial datum and thereby all the computations can be done in parallel to produce detailed global stability behavior and the resulting basins of attraction. With the aid of highly parallel Connection Machines, we were able to detect a wealth of the detailed nonlinear behavior of these schemes which would have been overlooked had isolated initial data been chosen on the CrayYMP or other serial or vector machine.

\section{Outline:}

The outline of this paper is as follows. Section 3 reviews background material. Section 4 describes the three $2 \times 2$ systems of nonlinear first-order autonomous model ODEs. Section 5 describes the 
four implicit LMM time discretizations and the four commonly used noniterative and iterative procedures in solving the resulting nonlinear algebraic equations. Section 6 discusses the combined bifurcation diagrams and numerical basins of attraction of the underlying schemes for the three model equations. This section also includes the result of using straight Newton method to solve the steady part of the ODE $\frac{d U}{d t}=S(U)$ (i.e., solving for the solutions of $S(U)=0$ ). Due to the complexity of the subject matter most of our study used a fixed time step. A brief discussion on variable step size control will be included. The paper ends with a summary and a discussion of the implications of the numerical studies in Sec. 7. Our studies reveal that unconditionally stable LMMs perform better than standard explicit methods and that noniterative implicit schemes are more efficient and mimic more closely the true behavior of the governing equations than the iterative implicit procedure for all of the four studied implicit LMMs. Studies further show that standard variable time step control, although performing better with very restricted time steps than the constant time step case, did not alleviate completely the occurrence of spurious numerical asymptotes.

\section{Preliminaries}

Consider a $2 \times 2$ system of first-order autonomous nonlinear ODEs of the form

$$
\frac{d U}{d t}=S(U)
$$

where $U$ and $S$ are vector functions of dimension 2 , and $S(U)$ is nonlinear in $U$. A fixed point (steady state) $U_{E}$ of an autonomous system (3.1) is a constant solution of (3.1); that is

$$
S\left(U_{E}\right)=0,
$$

where the subscript "E" stands for "exact" and $U_{E}$ denotes the fixed points of the ODE as opposed to the additional fixed points of the discretized counterparts (spurious fixed points) due to the numerical methods which we will encounter later.

Consider a nonlinear discrete map from a finite discretization of (3.1)

$$
U^{n+1}=U^{n}+D\left(U^{n}, \Delta t\right)
$$

where $\Delta t$ is the time step and $D\left(U^{n}, \Delta t\right)$ is linear or nonlinear in $\Delta t$ depending on the nu- merical method. A fixed point $\bar{U}$ of (3.3) is defined by $U^{n+1}=U^{n}$, or

$$
\bar{U}=\bar{U}+D(\bar{U}, \Delta t)
$$

or

$$
D(\bar{U}, \Delta t)=0 .
$$

A fixed point $\bar{U}$ of period $p>0$ of (3.3) is defined by $U^{n+p}=U^{n}$ where $U^{n+k} \neq U^{n}$ for $k<p$. In the context of discrete systems, the term "fixed point" without indicating the period means "fixed point of period 1" or the steady-state solution of (3.3). Here, we use the term asymptote to mean a fixed point of any period, a limit cycle (invariant set), chaos, or a strange attractor.

The type of finite discretization of (3.1) represented in (3.3) assumed the use of two-time level schemes. Otherwise the vector dimension of (3.3) would be $2(k-1)$ instead of 2 where $k$ is the number of the time level of the scheme. Here, the vector function $D$ is assumed to be consistent with the ODE (3.1) in the sense that fixed points of the ODE are fixed points of the scheme; however, the reverse need not hold. Also, spurious asymptotes that are asymptotic numerical solutions of (3.3) but not (3.1) can exist, depending on the numerical method and $\Delta t$. It is these features, accompanied by other added dynamics, that cause the discretized counterparts of the underlying ODE to possess a much richer dynamical behavior than the original ODE which forms the core of this study. Thus, the fixed points $\bar{U}$ of $D(\bar{U}, \Delta t)=0$ may be true fixed points $U_{E}$ of (3.1) or spurious fixed points $U_{S}$. The spurious fixed points $U_{S}$ are not roots of $S(U)=0$. That is, $S\left(U_{S}\right) \neq 0$.

Letting $U^{n}=\bar{U}+\delta^{n}$, then a perturbation analysis on (3.3) by discarding terms of $O\left(\delta^{2}\right)$ yields

$$
\delta^{n+1}=\left(I+\frac{\partial D(\bar{U}, \Delta t)}{\partial U}\right)^{n+1} \delta^{0} .
$$

Assuming $\frac{\partial D(\bar{U}, \Delta t)}{\partial U} \neq 0$, then the fixed point $\bar{U}$ is stable if the eigenvalues of $I+\frac{\partial D(\bar{U}, \Delta t)}{\partial U}$ lie inside the unit circle. If both eigenvalues are real and both lie inside (outside) the unit circle, then the fixed point is a stable (unstable) node. If one is inside the unit circle and the other outside, then the fixed point is a saddle. If both eigenvalues are complex, then the fixed point is a spiral. If the eigenvalues lie on the unit circle, then the fixed point of (3.3) is indeterminant and additional analysis is required 
to determine the true behavior of (3.3) around this type of fixed point. For a more refined definition and the difference in fixed point definition between ODEs and discrete maps, see Guckenheimer \& Holmes [1983] and Hale \& Kocak [1991] and references cited therein.

An important feature which can arise (for both systems of ODEs (3.1) and their discretizations) as the result of a Hopf bifurcation is a limit cycle where the trajectory traverses a closed curve in phase space. In all but a few simple cases, such limit cycles are beyond analysis. It is possible that the ODEs posses no limit cycle, but depending on the numerical methods, spurious limit cycles can be present for the discretized counterparts. It is this phenomena that can contribute to the nonconvergence of numerical schemes in timedependent approaches to the steady states.

\section{Model Nonlinear First-Order Autonomous ODEs}

Three of the four $2 \times 2$ systems of nonlinear firstorder autonomous model ODEs considered in Yee $\&$ Sweby [1992] are considered here. As before, we do not treat any system parameter present in the DEs as a bifurcation parameter, but instead keep it constant throughout each numerical calculation so that only the discretization parameters come into play. The systems considered with $U^{T}=(u, v)$ or $z=u+i v$ are a

1. Dissipative complex model:

$$
\frac{d z}{d t}=z\left(i+\varepsilon-|z|^{2}\right)
$$

2. Predator-Prey model:

$$
\begin{aligned}
& \frac{d u}{d t}=-3 u+4 u^{2}-0.5 u v-u^{3} \\
& \frac{d v}{d t}=-2.1 v+u v
\end{aligned}
$$

\section{Perturbed Hamiltonian system model:}

$$
\begin{aligned}
& \frac{d u}{d t}=\varepsilon(1-3 u)+\frac{3}{4}\left[1-2 u+u^{2}-2 v(1-u)\right] \\
& \frac{d v}{d t}=\varepsilon(1-3 v)-\frac{3}{4}\left[1-2 v+v^{2}-2 u(1-v)\right] .
\end{aligned}
$$

Here, $\varepsilon$ is the system parameter for (4.1) and (4.3).
The perturbed Hamiltonian model can be related to the numerical solution of the viscous Burgers' equation with no source term:

$$
\frac{\partial u}{\partial t}+\frac{1}{2} \frac{\partial\left(u^{2}\right)}{\partial x}=\beta \frac{\partial^{2} u}{\partial x^{2}} \quad \beta>0
$$

Let $u_{j}(t)$ represent an approximation to $u\left(x_{j}, t\right)$ of (4.4) where $x_{j}=j \Delta x, j=1, \ldots, J$, with $\Delta x$ the uniform grid spacing. Consider the three-point central difference spatial discretization with periodic condition $u_{J+j}=u_{j}$, and assume $\sum_{j=1}^{J} u_{j}=$ constant, which implies that $\sum_{j=1}^{J} \frac{d u_{j}}{d t}=0$. If we take $J=3$ and $\Delta x=1 / 3$, then, with $\varepsilon=9 \beta$ this system can be reduced to the $2 \times 2$ system of first-order nonlinear autonomous ODEs (4.3) with $U^{T}=\left(u_{1}, u_{2}\right)=(u, v)$. In this case, the nonlinear convection term is contributing to the nonlinearity of the ODE system (4.3).

These three equations were selected to bring out the dynamics of numerics for three different types of solution behavior of the nonlinear ODEs. The dissipative complex system (4.1) possesses either a unique stable fixed point or limit cycle with an unstable fixed point depending on the value of $\varepsilon$. This is the rare situation where the analytical expression of a limit cycle can be found. The predator-prey model (4.2) exhibits multiple stable fixed points. The perturbed Hamiltonian model (4.3), which arises as a gross simplification of the finite discretization of the viscous Burgers' equation, exhibits a unique stable fixed point. Following the classification of fixed points of (3.1) in Sec. 3, one can easily obtain the following:

Fixed Points of (4.1):

The dissipative complex model has a unique fixed point at $(u, v)=(0,0)$ for $\varepsilon \leq 0$. The fixed point is a stable spiral if $\varepsilon<0$ and a center if $\varepsilon=0$. For $\varepsilon>0$, the fixed point $(0,0)$ becomes unstable with the birth of a stable limit cycle with radius equal to $\sqrt{\varepsilon}$ centered at $(0,0)$. Figure 4.1 shows the phase portrait ( $u-v$ plane) of system (4.1) for $\varepsilon=-1$ and $\varepsilon=1$, respectively. Here, the entire $(u, v)$ plane belongs to the basins of attraction of the stable fixed point $(0,0)$ if $\varepsilon<0$. On the other hand, if $\varepsilon>0$, the entire $(u, v)$ plane except the unstable fixed point $(0,0)$ belongs to the basins of attraction of the stable limit cycle. 


\section{Phase Portraits \& Basins of Attraction Dissipative Complex Equation}
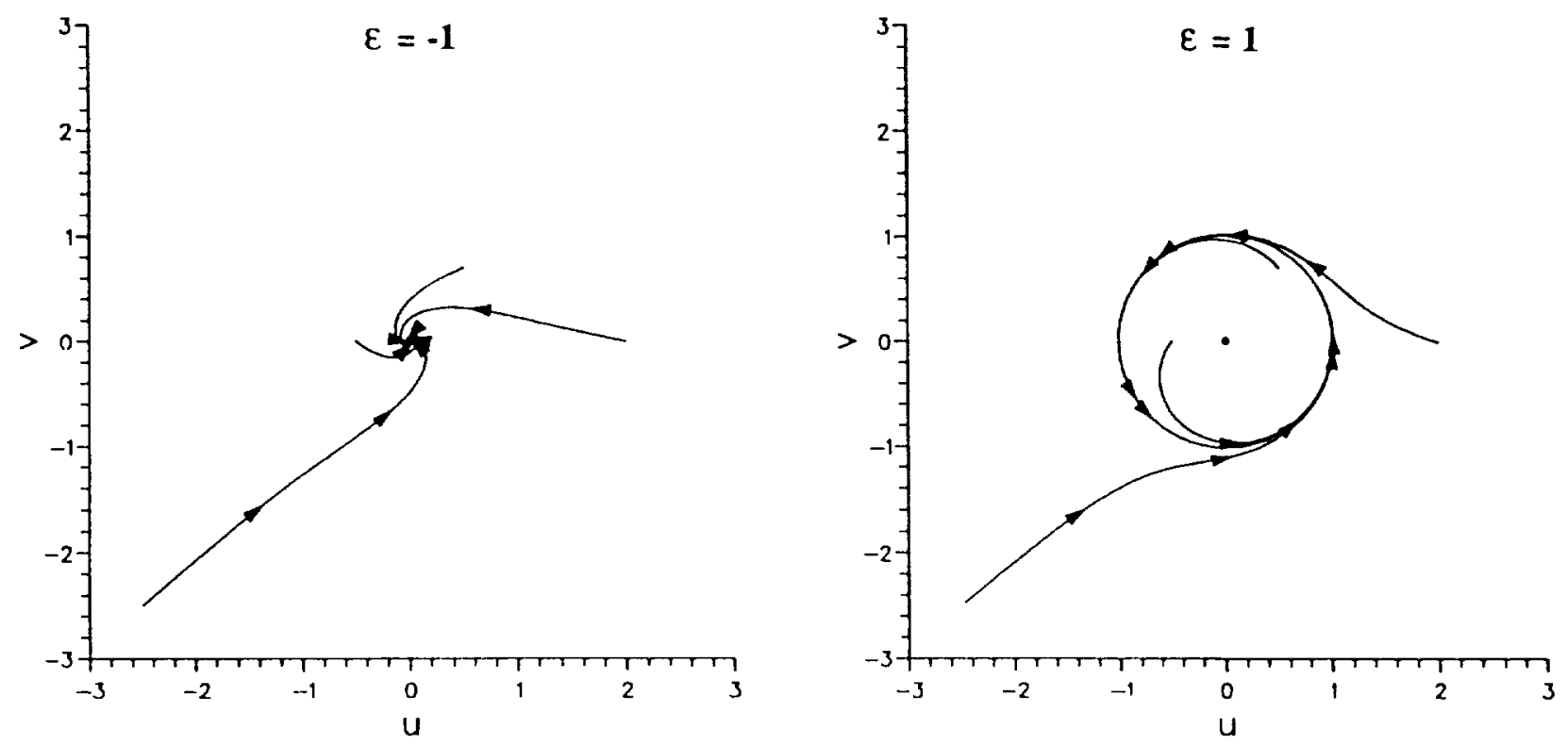

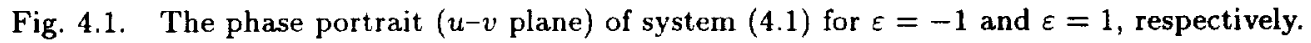

\section{Phase Portrait \& Basins of Attraction Predator-Prey Equation}

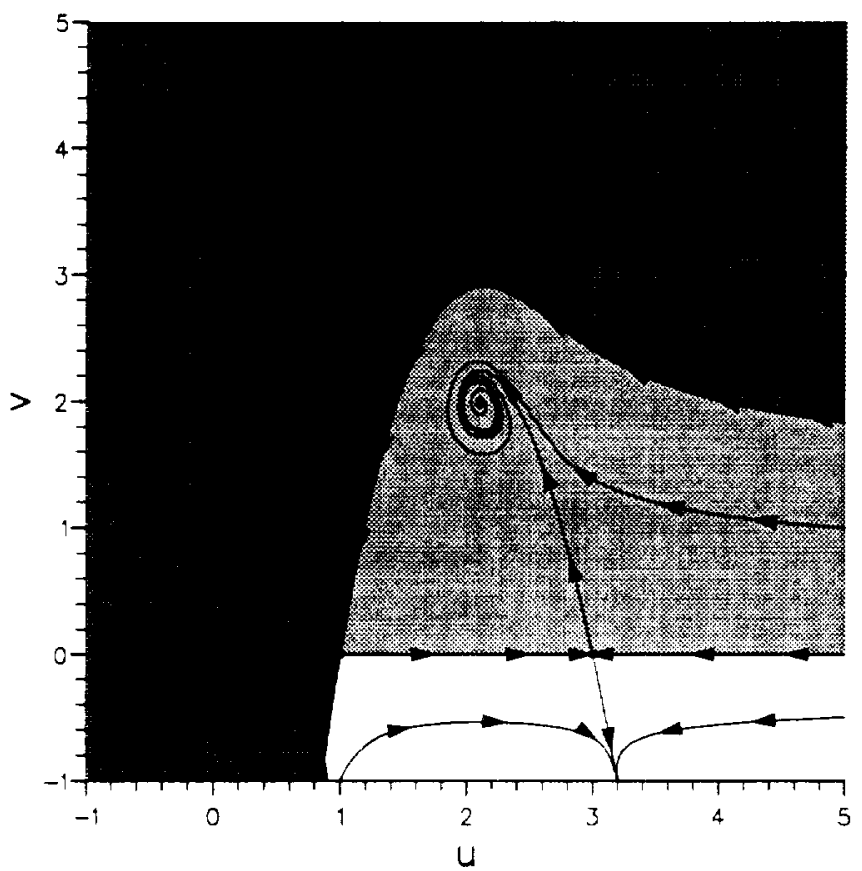

Fig. 4.2. The phase portrait and their corresponding basins of attraction for system (4.2).
Fixed Points of (4.2):

The predator-prey equation has four fixed points $(0,0),(1,0),(3,0)$, and $(2.1,1.98)$. By looking at the eigenvalues of the Jacobian of $S$, one finds that $(0,0)$ is a stable node, $(2.1,1.98)$ is a stable spiral, and $(1,0)$ and $(3,0)$ are saddles. Figure 4.2 shows the phase portrait and their corresponding basins of attraction for system (4.2). The different shades of grey regions represent the various basins of attraction of the respective stable fixed points. The white region represents the basin of divergent solutions. Note that the trajectories near the unstable separatrices actually do not merge with the unstable branch of separatrices, but only appear to merge due to the thick drawings of the solution trajectories.

\section{Fixed Points of (4.3):}

The perturbed Hamiltonian (semidiscrete system of the viscous Burgers' equation with three-point central difference in space) has four steady-state solutions of which three are saddles and one is a stable spiral at $(1 / 3,1 / 3)$ for $\varepsilon>0$. For $\varepsilon=0$, the stable spiral becomes a center. Figure 4.3 shows 


\section{Phase Portraits \& Basins of Attraction Viscous Burger's Equation (Central Difference in Space)}
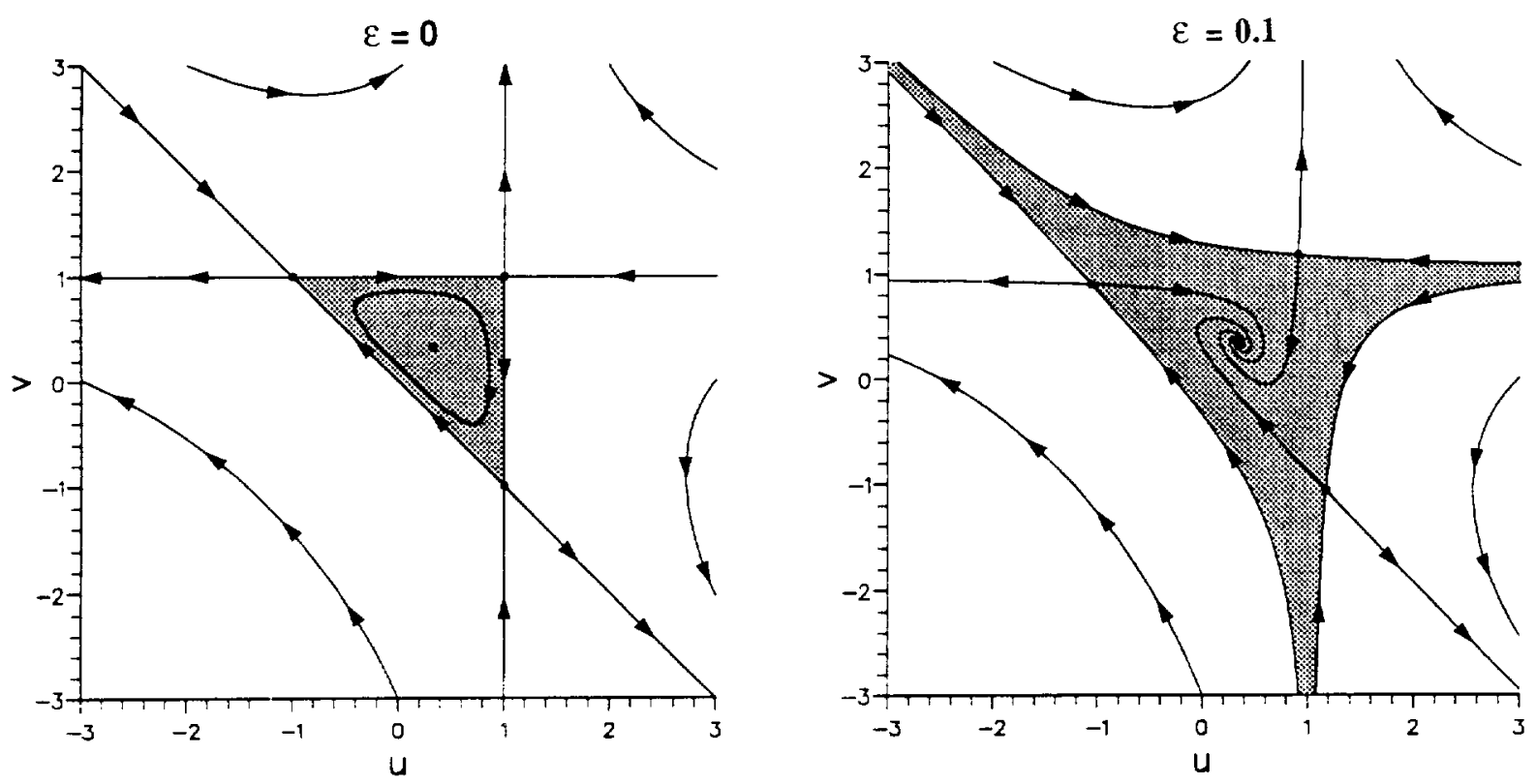

Fig. 4.3. The phase portrait and their corresponding basins of attraction for system (4.3).

the phase portrait and their corresponding basins of attraction for system (4.3). The shaded region represents the basins of attraction for the fixed point $(1 / 3,1 / 3)$ for $\varepsilon=0$ and $\varepsilon=0.1$. The white region represents the basin of divergent solutions. From here on we refer to (4.3) also as a viscous Burgers' equation with central difference in space.

\section{Numerical Methods}

The four LMMs for (3.1) considered here are

\section{Implicit Euler method}

$$
U^{n+1}=U^{n}+\Delta t S^{n+1}
$$

\section{Trapezoidal method}

$$
U^{n+1}=U^{n}+\frac{1}{2} \Delta t\left(S^{n}+S^{n+1}\right)
$$

3. 3-Level backward differentiation formula (BDF)

$$
U^{n+1}=U^{n}+\frac{2}{3} \Delta t S^{n+1}+\frac{1}{3}\left(U^{n}-U^{n-1}\right)
$$

4. Mid-point implicit method (one-legged trapezoidal)

$$
U^{n+1}=U^{n}+\Delta t S\left[\frac{1}{2}\left(U^{n+1}+U^{n}\right)\right] .
$$

Performing standard perturbation analysis on the above equations at the fixed points $\bar{U}$ of the ODE system by writing $U^{n}=\bar{U}+\delta^{n}$ where $S(\bar{U})=0$ and discarding terms of $O\left(\delta^{2}\right)$ yields

$$
\delta^{n+1}=K(\bar{U}) \delta^{n}
$$

where the matrix $K(\bar{U})$ is defined implicitly for the 3-level BDF method. The stability of the perturbation is therefore governed by the eigenvalues $\mu$ of the matrix $K(\bar{U})$.

For the implicit Euler method we find that

$$
K(\bar{U})=[I-\Delta t J(\bar{U})]^{-1},
$$

where $J(\bar{U})$ is the Jacobian $d S / d U$ evaluated at the fixed point, while for the trapezoidal and midpoint implicit methods

$$
K(\bar{U})=\left[I-\frac{1}{2} \Delta t J(\bar{U})\right]^{-1}\left[I+\frac{1}{2} \Delta t J(\bar{U})\right],
$$


and for the 3-level BDF method we have the relationship

$$
\left[I-\frac{2}{3} \Delta t J(\bar{U})\right] K(\bar{U})^{2}-\frac{4}{3} K(\bar{U})+\frac{1}{3} I=0 .
$$

Thus, if the eigenvalues of $J(\bar{U})$ are $\lambda$, we have the eigenvalues $\mu$ of $K(\bar{U})$ as follows:

$$
\begin{aligned}
\text { Implicit Euler } & \mu=\frac{1}{1-\Delta t \lambda}, \\
\text { Trapezoidal \& } & \mu=\frac{2+\Delta t \lambda}{2-\Delta t \lambda}, \\
\text { idpoint Implicit } & \\
\text { 3-level BDF } & \mu=\frac{1}{2 \mp \sqrt{1+2 \Delta t \lambda}},
\end{aligned}
$$

with both matrices sharing the same eigenvectors. (Note that since the expression (5.8) is a quadratic in $K(\bar{U})$ there are four possible modes corresponding to the different eigenvalues $\lambda$ and the two possible signs of the square root. However, it is the modes taking the negative square root which have larger modulus and therefore govern stability of the perturbation.)

The stability of the corresponding fixed points based on the eigenvalues of $K(\bar{U})$ can be determined exactly and were used to check our numerical computations later. They are summarized in Table 1. When numerically computing the full discretized equations there are various options which can be used to solve Eqs. (5.1), (5.2), or (5.3). We consider here linearization (a noniterative procedure /Yee \& Sweby, 1992]), simple iteration, Newton iteration, and modified Newton iteration.

Linearization is achieved by expanding $S^{n+1}$ as $S^{n}+J\left(U^{n}\right)\left(U^{n+1}-U^{n}\right)$. Thus, the linearized implicit Euler method is

$$
U^{n+1}=U^{n}+\Delta t\left[I-\Delta t J\left(U^{n}\right)\right]^{-1} S^{n},
$$

the Linearized Trapezoidal method (and also the linearized midpoint implicit method) is

$$
U^{n+1}=U^{n}+\Delta t\left[I-\frac{1}{2} \Delta t J\left(U^{n}\right)\right]^{-1} S^{n},
$$

and the Linearized 3-level BDF method is

$$
\begin{aligned}
U^{n+1}= & U^{n}+\left[I-\frac{2}{3} \Delta t J\left(U^{n}\right)\right]^{-1} \\
& \times\left[\frac{2}{3} \Delta t S^{n}+\frac{1}{3}\left(U^{n}-U^{n-1}\right)\right] .
\end{aligned}
$$

Note that all three have possible singularities.
Straightforward perturbation analysis shows that while the matrices $K(\bar{U})$ for the perturbed form of the implicit scheme differ from the fully implicit schemes, their eigenvalues are the same. Note, however, that the dynamics away from the fixed points need not be identical since the perturbation analysis represents only the local behavior of hypberbolic fixed points of the unperturbed systems.

Simple Iteration is the process in which, given a scheme of the form

$$
U^{n+1}=G\left(U^{n}, U^{n+1}\right),
$$

we perform the iteration

$$
U_{(\nu+1)}^{n+1}=G\left(U^{n}, U_{(\nu)}^{n+1}\right) \quad \nu=1, \ldots
$$

where $U_{0}^{n+1}=U^{n}$ and " $(\nu)$ " indicates the iteration index. The iteration is continued either until some tolerance between iterates is achieved, i.e.,

$$
\left\|U_{(\nu+1)}^{n+1}-U_{(\nu)}^{n+1}\right\| \leq \text { tol }
$$

or a limiting number of iterations, in our case 15 , have been performed. The major drawback with simple iteration is that for guaranteed convergence the iteration must be a contraction, i.e.,

$$
\left\|G\left(U^{n}, V\right)-G\left(U^{n}, W\right)\right\| \leq \alpha\|V-W\|,
$$

where $\alpha<1$. Whether or not the iteration is a contraction at the fixed points will influence the stability of that fixed point, overriding the stability of the implicit scheme. Away from the fixed points the influence will be on the basins of attraction. For the four LMMs at the fixed points this translates as follows:

$$
\text { Implicit Euler } \Delta t\|J(\bar{U})\| \leq \alpha<1,
$$

$\begin{array}{r}\text { Trapezoidal \& } \\ \text { idpoint Implicit }\end{array} \frac{1}{2} \Delta t\|J(\bar{U})\| \leq \alpha<1$,

$$
\text { 3-level BDF } \frac{2}{3} \Delta t\|J(\bar{U})\| \leq \alpha<1 .
$$

As we shall see in Secs. 6 and 7, our numericall results illustrate this limitation well.

Newton Iteration for the implicit schemes is of the form

$$
\begin{aligned}
U_{(\nu+1)}^{n+1}=U_{(\nu)}^{n+1}-F^{\prime}\left(U^{n},\right. & \left.U_{(\nu)}^{n+1}\right)^{-1} \\
& \times F\left(U^{n}, U_{(\nu)}^{n+1}\right),
\end{aligned}
$$


Table 1. Stability of the fixed points of the perturbed discretized equations.

\begin{tabular}{cllll}
\hline System/Fixed Point & Exact & Implicit Euler & Trap & BDF \\
\hline $\begin{array}{c}\text { Dissipative Complex } \\
(0,0) \varepsilon<0\end{array}$ & SS & SS & SS & Stable \\
$(0,0) \varepsilon>0$ & US & US $\left(0, \frac{2 \varepsilon}{1+\varepsilon^{2}}\right)$ & US & Unstable $(0, ?)$ \\
& & SS $\left(\frac{2 \varepsilon}{1+\varepsilon^{2}}, \infty\right)$ & & Stable $(?, \infty)$ \\
$\begin{array}{c}\text { Predator Prey } \\
(0,0)\end{array}$ & SN & SN & SN & Stable \\
$(1,0)$ & S & S $(0,1)$ & S & Unstable $(0,2)$ \\
$(3,0)$ & SN $(1, \infty)$ & S & Stable $(2, \infty)$ \\
$(2.1,1.98)$ & S $\left(0, \frac{2}{0.9}\right)$ & S & Unstable $\left(0, \frac{4}{0.9}\right)$ \\
Perturbed Hamiltonian & & SN $\left(\frac{2}{0.9}, \infty\right)$ & & Stable $\left(\frac{4}{0.9}, \infty\right)$ \\
$\left(\frac{1}{3}, \frac{1}{3}\right) \varepsilon>0$ & SS & SS & SS & Stable \\
\hline
\end{tabular}

Key: SN - Stable Node, SS - Stable Spiral, US - Unstable Spiral, S - Saddle, Trap - trapezoidal, $\gamma=\varepsilon+\sqrt{\varepsilon^{2}+4}$. Stable/Unstable indicates that type could not be determined. Intervals indicate ranges of $\Delta t$ if not $(0, \infty)$.

where $U_{0}^{n+1}=U^{n}$. The differentiation is with respect to the second argument and the scheme has been written in the form (for two-time level schemes)

$$
F\left(U^{n}, U^{n+1}\right)=0 \text {. }
$$

Modified Newton Iteration is the same as (5.18) except it uses a frozen Jacobian $F^{\prime}\left(U^{n}, U^{n}\right)$. The same tolerance and maximum number of iterations used for the simple iteration are also used for the Newton and modified Newton iterations. In all of the computations, the starting scheme for the 3-level BDF is the linearized implicit Euler.

We also considered two variable time step control methods. The first one is "implicit Euler + Newton iteration with Local Truncation Error Control" [Dieci \& Estep, 1990]

$$
\begin{aligned}
U_{(0)}^{n+1}= & U^{n} \\
U_{(\nu+1)}^{n+1}= & U_{(\nu)}^{n+1}+\left[I-\Delta t^{n} J\left(U_{(\nu)}^{n+1}\right)\right]^{-1} \\
& \times\left[U_{(\nu)}^{n+1}-U^{n}-\Delta t^{n} S\left(U_{(\nu)}^{n+1}\right)\right] \quad \nu=1, \ldots
\end{aligned}
$$

with

$$
\begin{aligned}
\Delta t^{n}= & 0.9 \Delta t^{n-1} \\
& \times \sqrt{\frac{\text { tol }_{1}}{\left\|U^{n}-U^{n-1}-\Delta t^{n-1} S\left(U^{n}\right)\right\|}},
\end{aligned}
$$

where the $(n+1)$ th step is rejected if $\| U^{n}-$ $U^{n-1}-\Delta t^{n-1} S\left(U^{n}\right) \|>2$ tol $_{1}$. In this case, we set $\Delta t^{n-1}=\Delta t^{n}$. The value "tol 1 " is a prescribed tolerance and the norm is an infinity norm. The second one is the popular "ode23" method

$$
\begin{aligned}
k_{1} & =S\left(U^{n}\right), \\
k_{2} & =S\left(U^{n}+\Delta t^{n} k_{1}\right), \\
k_{3} & =S\left(U^{n}+\Delta t^{n}\left(k_{1}+k_{2}\right) / 4\right), \\
U^{n+1} & =U^{n}+\Delta t^{n}\left(k_{1}+k_{2}+4 k_{3}\right) / 6, \\
\Delta U^{n+1} & =\Delta t^{n}\left(k_{1}+k_{2}-2 k_{3}\right) / 3,
\end{aligned}
$$

with

$$
\Delta t^{n}=0.9 \Delta t^{n-1} \sqrt{\frac{\text { tol }_{1}}{\left\|\Delta U^{n}\right\|}},
$$


where the $(n+1)$ th step is rejected if $\left\|\Delta U^{n+1}\right\|>$ tol $_{1} \max \left\{1,\left\|U^{n+1}\right\|\right\}$. In that case, we set $\Delta t^{n-1}=$ $\Delta t^{n}$. Again, "toll" is a prescribed tolerance and the norms are infinity norms.

We also employ "straight" Newton's method in solving for the solutions of $S(U)=0$ which is the one-step Newton iteration of the implicit Euler method of (5.18).

\section{Numerical Results}

As mentioned before, the nature of our calculations requires thousands of iterations of the same equation with different ranges of initial data on a preselected $(u, v)$ domain and ranges of the discretized parameter space $\Delta t$. The NASA Ames CM-2 and CM-5 allow vast numbers (typically 65,536 ) of calculations to be performed in parallel and is ideal for our studies. Two different representations of the numerical basins of attraction are computed on the Connection Machines. One is bifurcation diagrams as a function of $\Delta t$ with numerical basins of attraction superimposed on a constant $v$ - or $u$-plane. The other is the numerical basins of attraction with the stable asymptotes superimposed on the phase plane $(u, v)$ with selected values of $\Delta t$.

To obtain a bifurcation diagram with numerical basins of attraction superimposed on the CM-2 or CM-5, the preselected domain of initial data on a constant $v$ - or $u$-plane and the preselected range of the $\Delta t$ parameter are divided into 512 equal increments. For the bifurcation part of the computations, with each initial datum and $\Delta t$, the discretized equations are preiterated $5,000-9,000$ steps before the next 6,000 iterations (more or less depending on the problem and scheme) are plotted. The preiterations are necessary in order for the solutions to settle to their asymptotic value. A high number of iterations are overlaid on the same plot in order to detect periodic orbits or invariant sets. The reader is reminded that with this method of computing the bifurcation diagrams, only the stable branches are obtained. While computing the bifurcation diagrams it is possible to overlay basins of attraction for each value of $\Delta t$ used. For the basins of attraction part of the computations with each value of $\Delta t$ used, we keep track of where each initial datum asymptotically approaches and color code them (as a vertical strip) according to the individual asymptotes. While efforts were made to match color coding of adjacent strips on the bifurcation diagram, it was not always practical or possible. Care must, therefore, be taken when interpreting these overlays. See Secs. 6.1 and 6.2 for discussions.

For the basins of attraction on the phase plane $(u, v)$ with selected values of $\Delta t$ and the stable asymptotes superimposed, the $(u, v)$ domain is divided into $512 \times 512$ points of initial datum. With each initial datum and $\Delta t$, we preiterate the respective discretized equation $5,000-9,000$ steps and plot the next 6,000 steps to produce the asymptotes (fixed points of various order and limit cycles). Again, for the basins of attraction part of the computations, for each value of $\Delta t$ used, we keep track of where each initial datum asymptotically approaches and color code them according to the individual asymptotes. Details of the techniques used for detection of asymptotes and basins of attraction are given in the appendix of Sweby \& Yee [1991]. Note that in all of the plots, if color printing is not available, the different shades of gray represent different colors.

Selected results for both representations of numerical basins of attraction are shown in Figs. 6.16.33. In the plots, $r=\Delta t$. The " $\mathrm{r}=\mathrm{aDt}$ " label denotes a scaling parameter " $a$ " (set to unity for calculations presented here) times the time step $\Delta t$. White dots and white curves on the basins of attraction with bifurcation diagrams superimposed represent the bifurcation curves. White dots and white closed curves on the basins of attraction with the numerical asymptotes superimposed represent the stable fixed points, stable periodic solutions, or stable limit cycles. The black regions represent divergent solutions.

Note that the preselected regions of $\Delta t$ and the selected $\Delta t$ for the phase diagrams for both representations of numerical basins of attraction were determined by examining a wide range of $\Delta t$. In most cases, we examined $\Delta t$ from close to zero up to one million. What are shown in Figs. 6.1-6.33 represent some of the $\Delta t$ ranges that are most interesting. Note also that for all of these models, using $\Delta t=0.1$ is approximately $1 / 10$ (or less) the time step limit of standard explicit methods [Yee \& Sweby, 1992].

The streaks on some of the plots are either due to the nonsettling of the solutions within the prescribed number of iterations or the existence of small isolated spurious asymptotes. Due to the high cost of computation, no further attempts were made 
to refine their detailed behavior since our purpose was to show how, in general, the different numerical methods behave in the context of nonlinear dynamics. From our numerical studies, the midpoint implicit method (linearized or iterative methods) behaves the same as, or very similarly to, the trapezoidal method for the three models. Thus, no figures will be shown of the midpoint implicit method. In general, the dynamics of implicit LMMs are very different from the dynamics of standard explicit methods. See reference [Yee \& Sweby, 1992] for the dynamics of nine explicit methods.

\subsection{Numerical results for the dissipative complex equation}

Figures 6.1-6.10 show selected results for the two representations of numerical basins of attraction for model (4.1) for $\varepsilon=1$. The exact solution for (4.1) with $\varepsilon=1$ is a stable limit cycle with unit radius centered at $(0,0)$. The "exact" basin of attraction for the limit cycle is the entire $(u, v)$ plane except the unstable fixed point $(0,0)$.

Figures 6.1-6.3 compare the noniterative with the iterative procedures for solving the nonlinear algebraic equations using implicit Euler, trapezoidal, and 3-level BDF methods. Figures 6.4-6.10 show selected results of the stable numerical asymptotes with basins of attraction superimposed using four different $\Delta t$ by the three implicit LMMs. The red regions are the numerical basins of attraction for the stable limit cycle except in Fig. 6.5 $(\Delta t=2.65)$, in Fig. $6.6(\Delta t=1.5,2,4)$, in Fig. 6.8 $(\Delta t=1.5)$, in Fig. $6.9(\Delta t=2,2.5,4)$, and in Fig. $6.10(\Delta t=1.515,2.5)$. The green regions shown in Figs. 6.1 and $6.3-6.5$ are the numerical basins of attraction for the stabilized fixed point $(0,0)$. Note how the implicit method turns the unstable fixed point $(0,0)$ of the ODE system into a stable one for $\Delta t \geq 1$.

To aid in the understanding of some of the results shown in Figs. 6.4-6.10, the following gives some explanation on how to interpret the basins of attraction diagrams with the stable numerical asymptotes superimposed. All of the selected time steps $\Delta t$ shown in Figs. 6.4-6.10 are based on Figs. 6.1-6.3 where the bifurcation diagrams with the basins of attraction are superimposed. These time steps were chosen to illustrate selected features of the different bifurcation phenomena on the $(u, v)$ plane.
For example, it is easier to understand Fig. 6.8 $(\Delta t=1.5)$ using "trapezoidal + modified Newton" if we look at the fourth plot in Fig. 6.2. Figure 6.2 shows that the original limit cycle bifurcates into a "period 2 type" limit cycle near $\Delta t=1.25$. Figure 6.2 shows distinctively that both rings share the same basin (only one distinct solid red basin of attraction). The lack of a solid basin of attraction in the third plot of Fig. 6.8 is due to the coloring algorithm, which requires a repetition of the limit cycle within the time of integration in order to distinguish basins of attraction. When this repetition is not present the resulting coloring gives a crude indication of trajectories. Additional preiteration steps (many more than 9,000 ) would likely alleviate the problem. Note that we need all of the trajectories corresponding to the $512 \times 512$ initial data to settle to within the prescribed preiterations before a solid basin results. The fourth plot of Fig. 6.8 together with Fig. 6.2 hints at a rapid period doubling transition to instability.

Figure 6.9 for $\Delta t=2$ illustrates failure of the coloring algorithm to detect the basin of attraction due to an insufficient number of preiterations. The result is again a crude indication of the trajectories. Even though not correctly colored, the non-black region gives the size of the basin. Also, the third plot of Fig. 6.3 gives a clear indication of the size of the corresponding numerical basin of attraction for $\Delta t=2$.

Figure 6.10 for $\Delta t=1.515$ illustrates the surprising presence of an embedded region of instability within the basin of attraction of the limit cycle (see also Fig. 6.3). The lack of a distinct (single red colored) basin is again an artifact of the coloring algorithm. Additional preiteration steps would likely alleviate this.

Figures 6.1, 6.3-6.6, and 6.8-6.10 illustrate the situation where unconditionally stable LMM schemes, such as the implicit Euler and 3-level BDF methods, can converge to a wrong solution if one picks the initial data inside the green region, even though this region contains valid physical initial data for the ODE. Thus, even though LMMs preserved the same number of fixed points as the underlying ODE, these fixed points can change type and stability. This phenomenon is related to the "nonrobustness" of implicit methods sometimes experienced in CFD computations. In these types of computations where the initial data are not known, the highest probability of avoiding spurious 


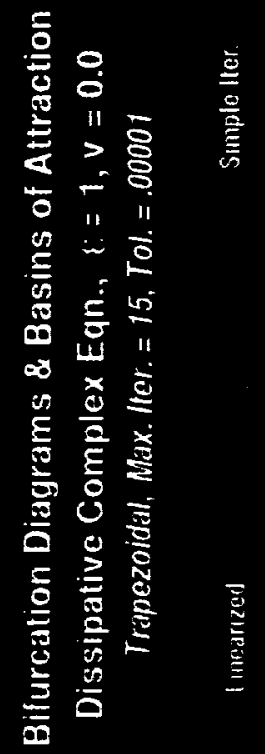

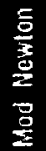

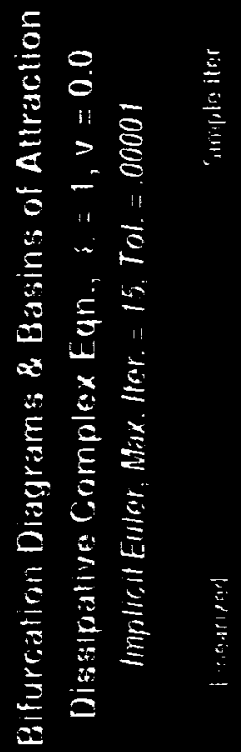

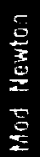



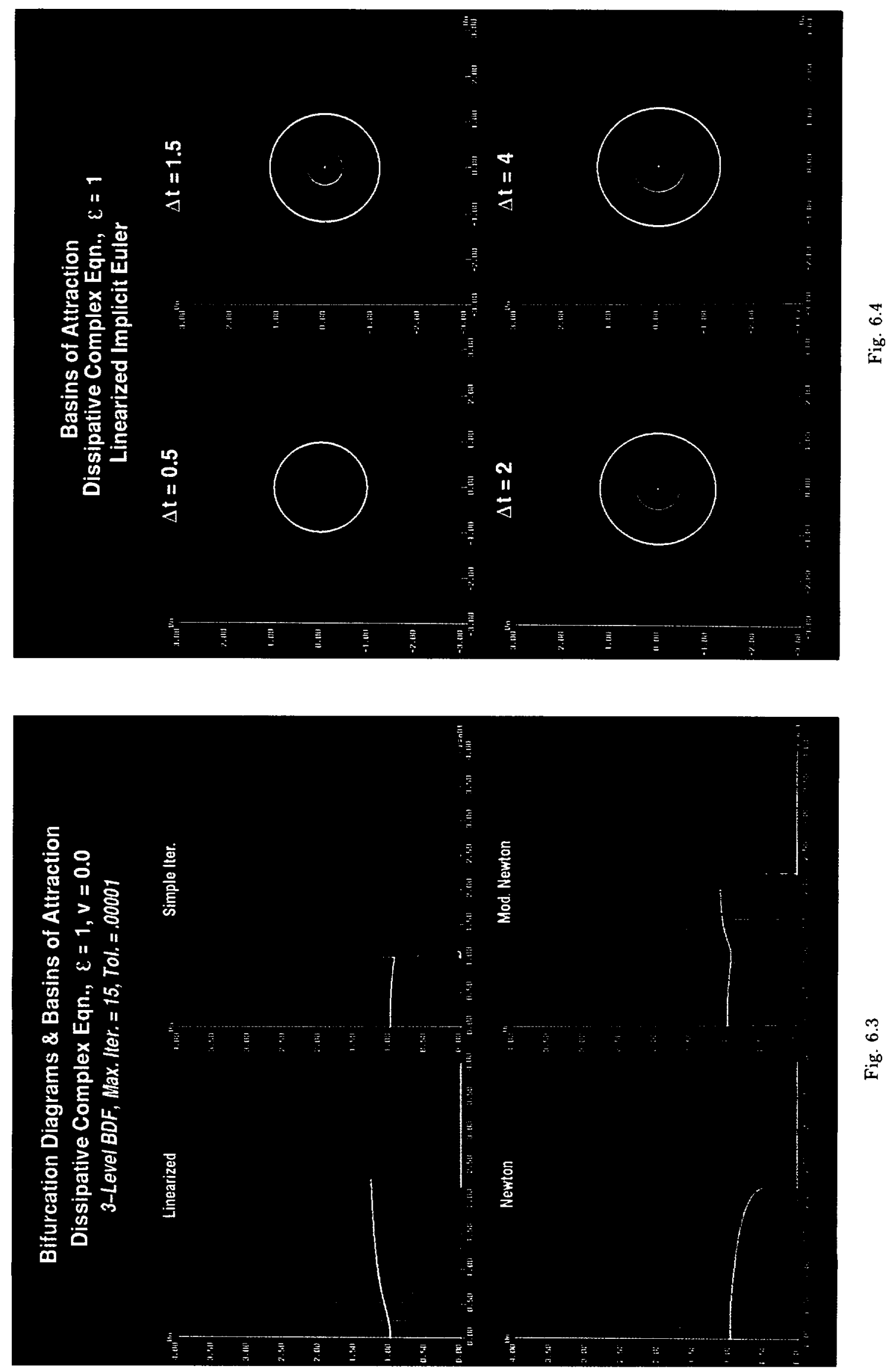

3

ف 

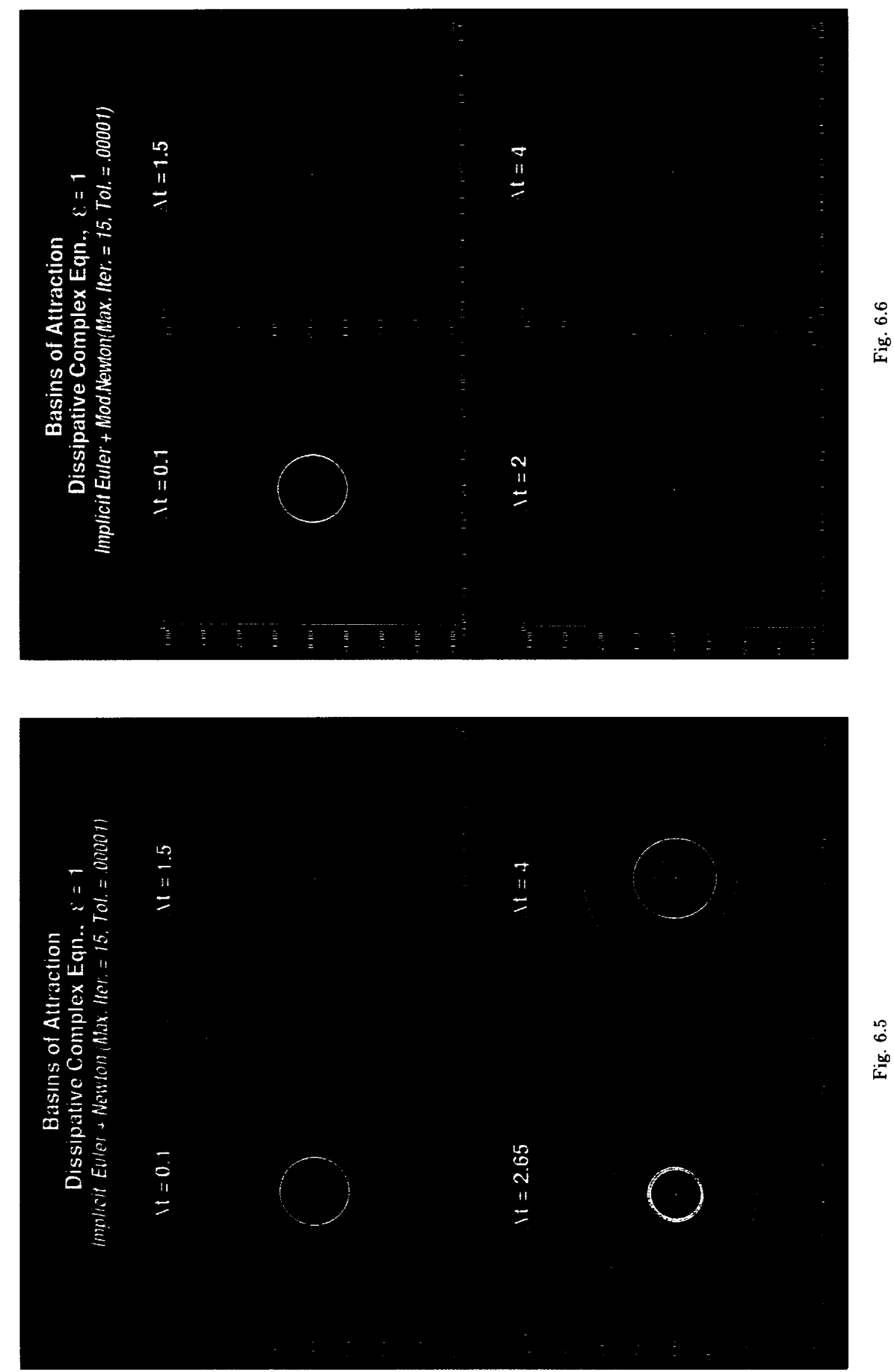

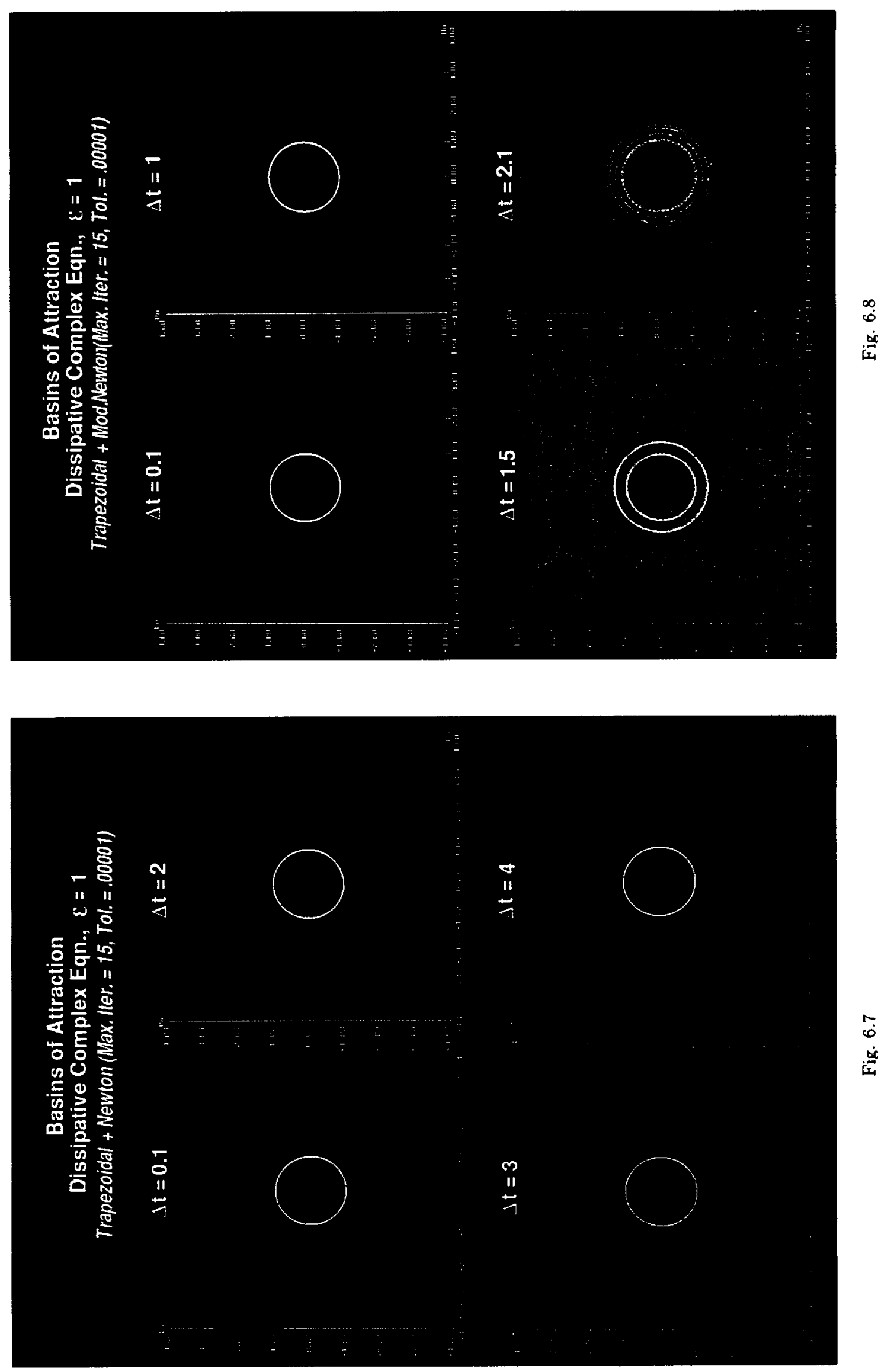

-
0
0 

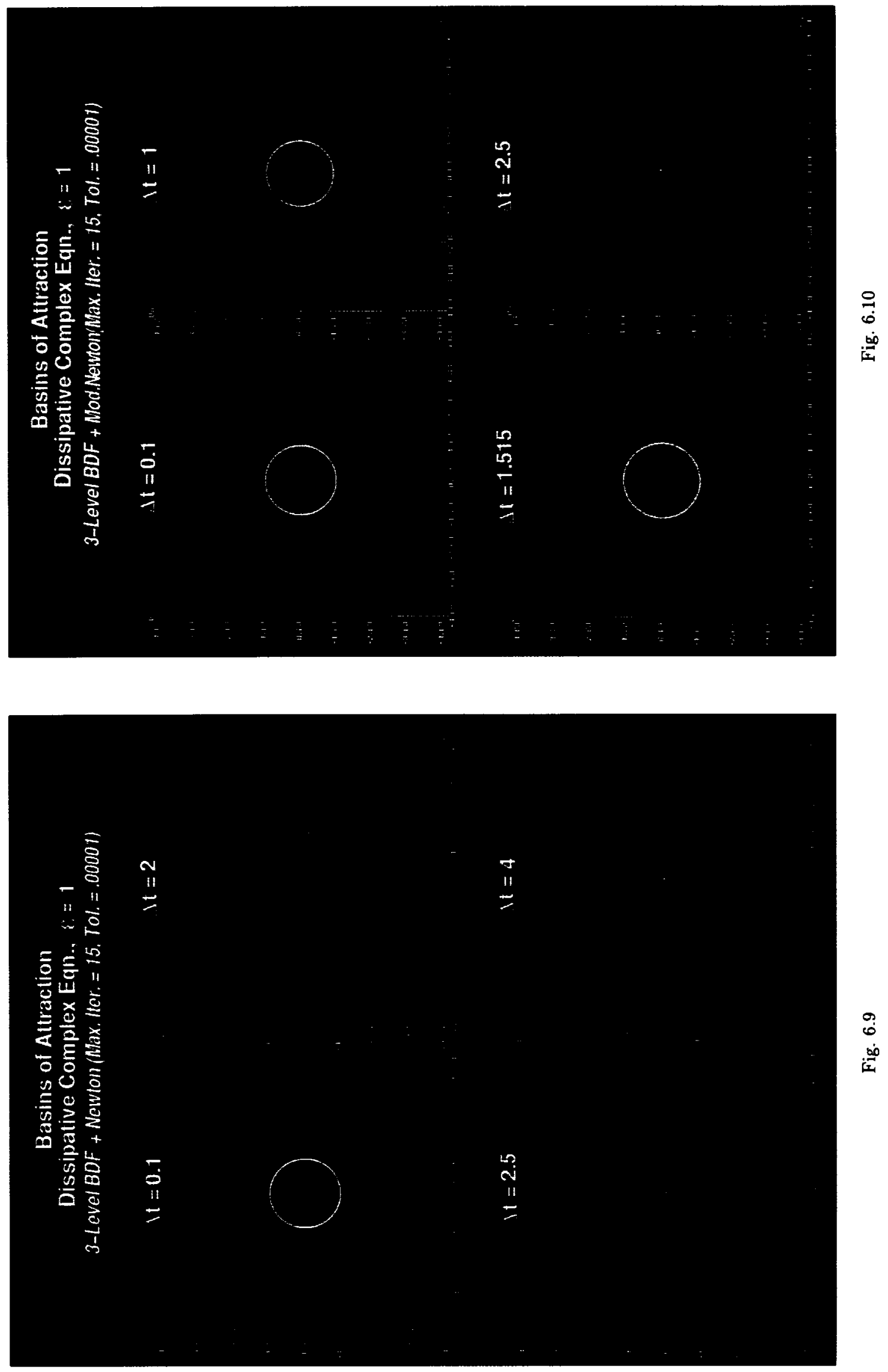
asymptotes is achieved when a fraction of the allowable linearized stability limit of $\Delta t$ is employed.

These diagrams also illustrate the unreliability of trying to compute a true limit cycle with any sizable $\Delta t$. This should not be surprising since the scheme only gives an $O\left(\Delta t^{p}\right)$ ( $p$ is the order of the scheme) approximation to the solution trajectories. In addition, since the limit cycle is not a fixed point, we would expect inaccuracies to be introduced. However, inaccuracies are not easy to detect in practice, especially when a numerical solution produces the qualitative features expected. Overall, the trapezoidal method and the midpoint implicit method (figures not shown) give more accurate solutions for the limit cycle. Note that all four LMMs except the implicit Euler are second-order accurate.

All of the four LMMs and four solution procedure combinations exhibit spurious stable and unstable asymptotes except spurious steady states. It is fascinating to see the dramatic difference in shapes and sizes of numerical basins of attraction for the different methods and solution procedure combinations compared with the exact basin of attraction. The evolution of the numerical basin of attraction as $\Delta t$ changes is very traumatic for all four LMMs and for the same LMM with different solution procedures. For larger $\Delta t$, the noniterative (linearized) implicit Euler, "implicit Euler + Newton", and "straight Newton" give the same numerical basins of attraction. Simple iteration behaves similarly to typical explicit methods (in terms of stability and the size of numerical basin of attraction) for all of the four studied implicit methods and for models (4.2) and (4.3) as well. The main advantage of the simple iteration procedure over nonLMM explicit methods is that spurious steady states cannot occur.

Numerical experiments were also performed on the dissipative complex model (4.1) using the adaptive time stepping strategies (5.20) and (5.21) based on local truncation error control. Our studies indicate that these two variable time step control implicit and explicit methods (5.20) and (5.21) can alleviate the spurious dynamics for most calculations. However, the allowable time step, determined by $(5.20)$ or $(5.21)$, is too small for practical usage, especially for the explicit method (5.21). For the implicit method (5.20), the allowable time step, determined by $(5.20)$, is slightly larger than the explicit method (5.21), but it is still impractical to use. Numerical experiments also indicated that with the wrong combination of starting time step, initial data and tolerance value, spurious dynamics could occasionally be produced.

\subsection{Numerical results for the predator-prey equation}

Selected results for the two representations of numerical basins of attraction for the predatorprey model (4.2) are shown in Figs. 6.11-6.22. Figures 6.11-6.13 compare the noniterative with the iterative procedures in solving the nonlinear algebraic equations using implicit Euler, trapezoidal, and 3-level BDF methods. Figures 6.14-6.22 show selected results of the numerical asymptotes with basins of attraction superimposed using four different $\Delta t$ s by the three implicit LMMs. Here, except for Figs. $6.14, \Delta t=0.4$ and 0.415 , the green regions represent the numerical basins of attraction for the stable spiral $(2.1,1.98)$ and red regions represent the numerical basins of attraction for the stable node $(0,0)$. The numerical basins of attraction in Figs. 6.17 and 6.18 with $\Delta t=0.1$ appear to be the same as the exact basins of attraction of the DE (4.2). The numerical basin of attraction by some of the LMMs for the fixed point $(0,0)$ is larger than the corresponding exact basin of attraction for the $\mathrm{DE}$ (4.2). (See Figs. 6.15, and 6.16 when $\Delta t=0.1$.) In this case, the numerical basins of attraction for the divergent solution (black region) is smaller than the true one. The dramatic difference in shapes and sizes of numerical basins of attraction for the different methods and solution procedure combinations compared with the exact basin of attraction is even more fascinating than the dissipative complex model.

All four LMMs and solution procedure combinations, other than simple iteration, change the two saddle points into stable or unstable fixed points of other types as illustrated in Figs. 6.14-6.22. For the implicit Euler, the two fixed points $(2.1,1.98)$ and $(0,0)$ are unconditionally stable and the stabilized fixed points $(1,0)$ and $(3,0)$ (saddles for the original ODE) are almost unconditionally stable except for small $\Delta t$. This is most interesting in the sense that the numerical basins of attraction for the stable exact fixed points $U_{E}$ of the model (4.2) by the implicit Euler method were permanently altered for $\Delta t$ near or larger than 3, as illustrated in Figs. 6.156.17 . It would be easier to interpret the results in Fig. 6.11 if one interchanged the yellow and green colors for $\Delta t \geq 1$. Our studies also indicated that 


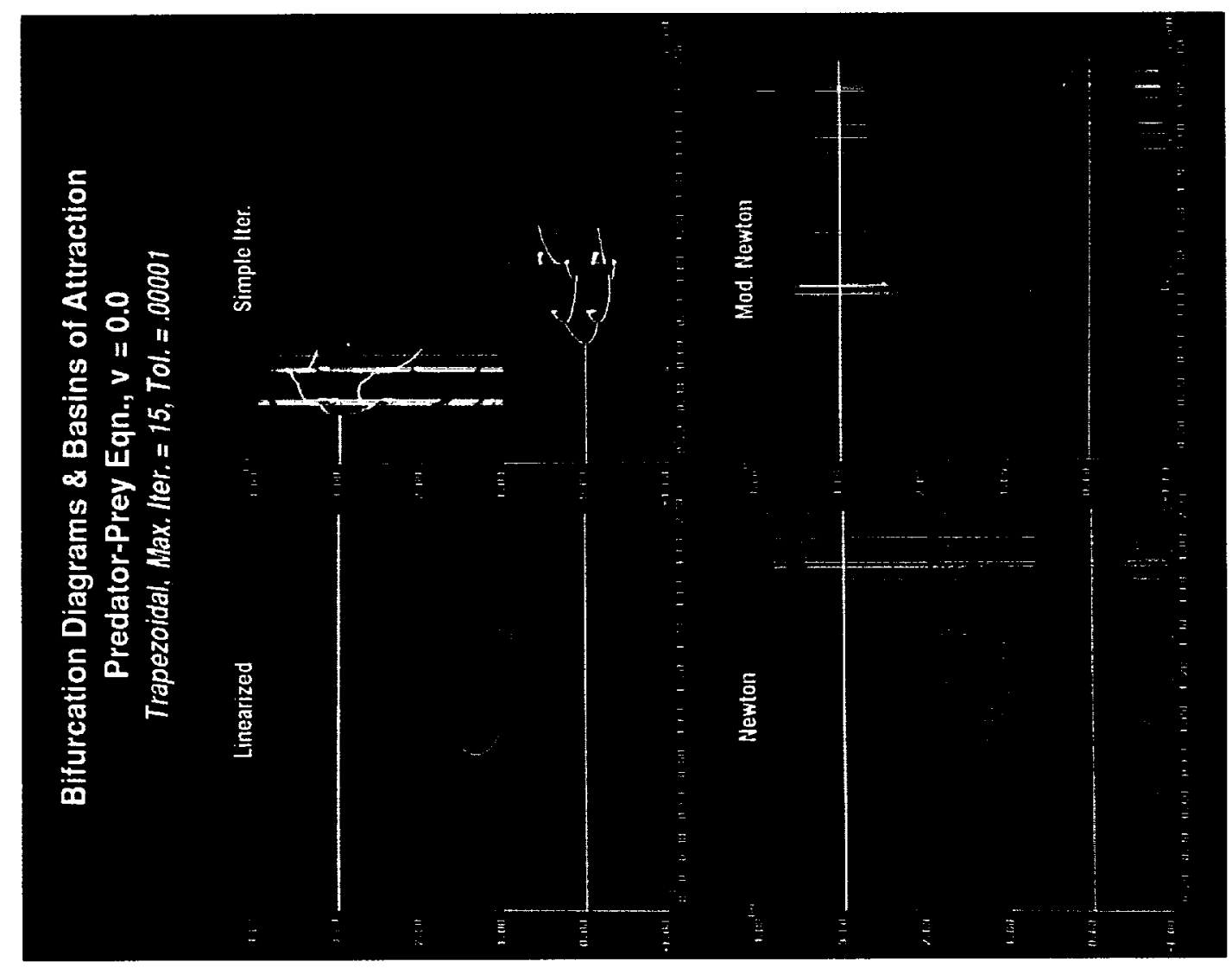

7
6
00
01

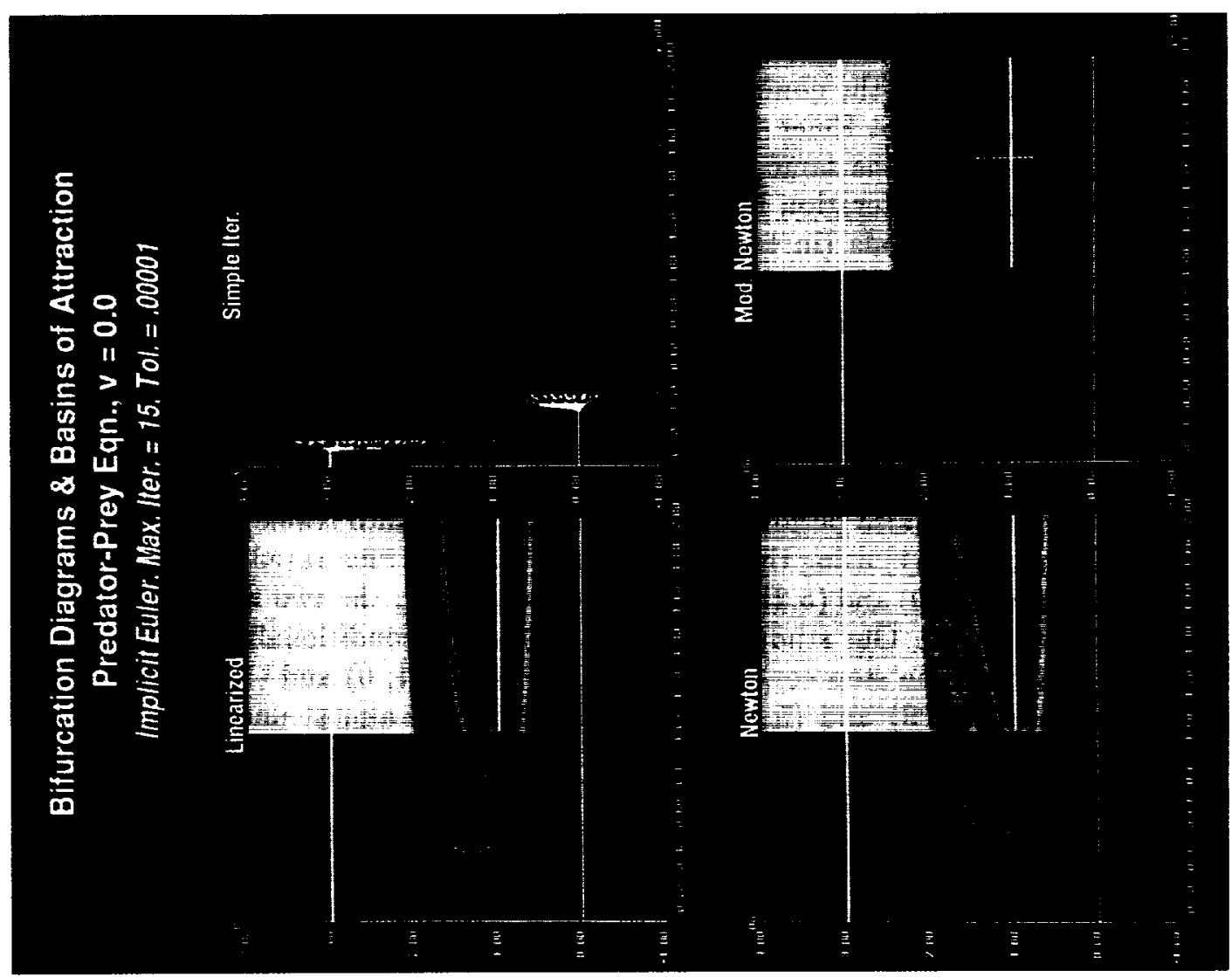

7
$\dot{0}$
$\dot{10}$ 


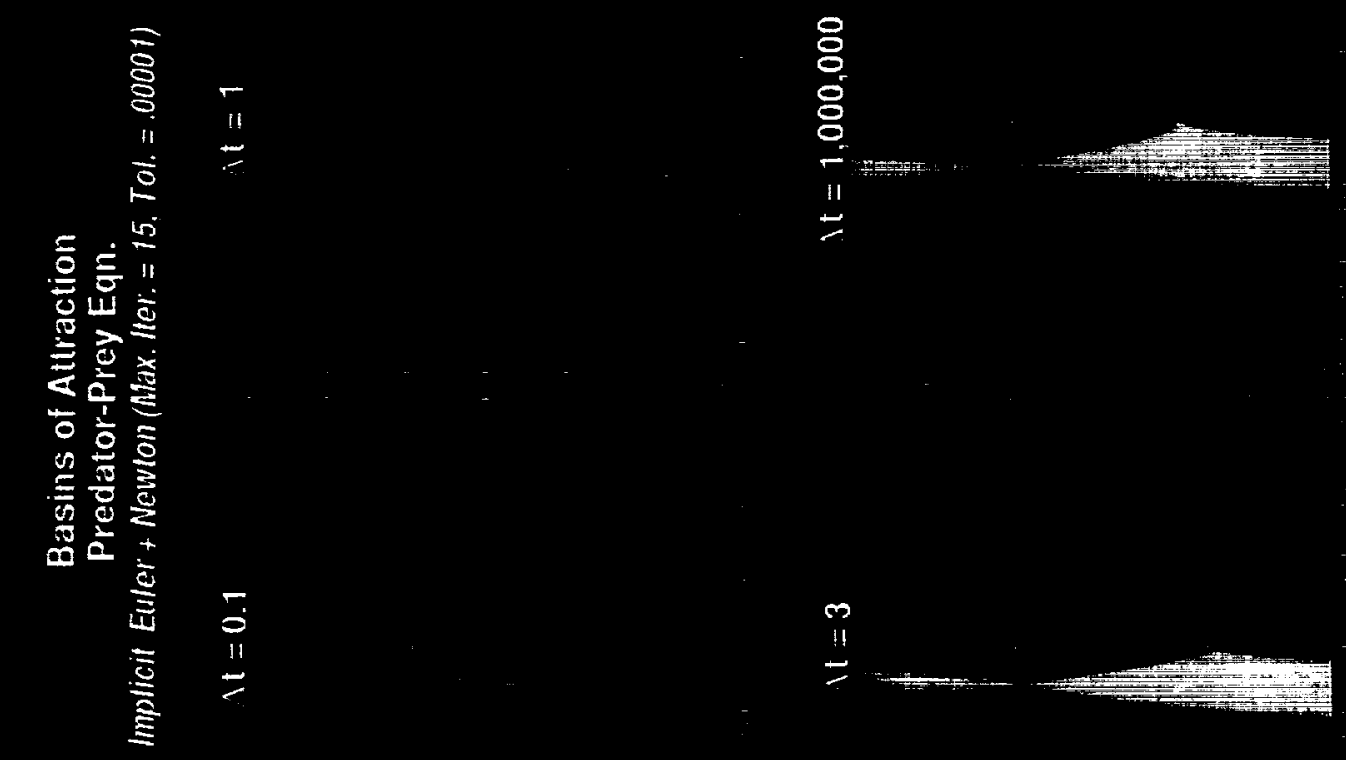

8

$\pm$

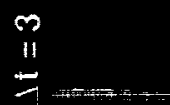

$\ldots+1$. 

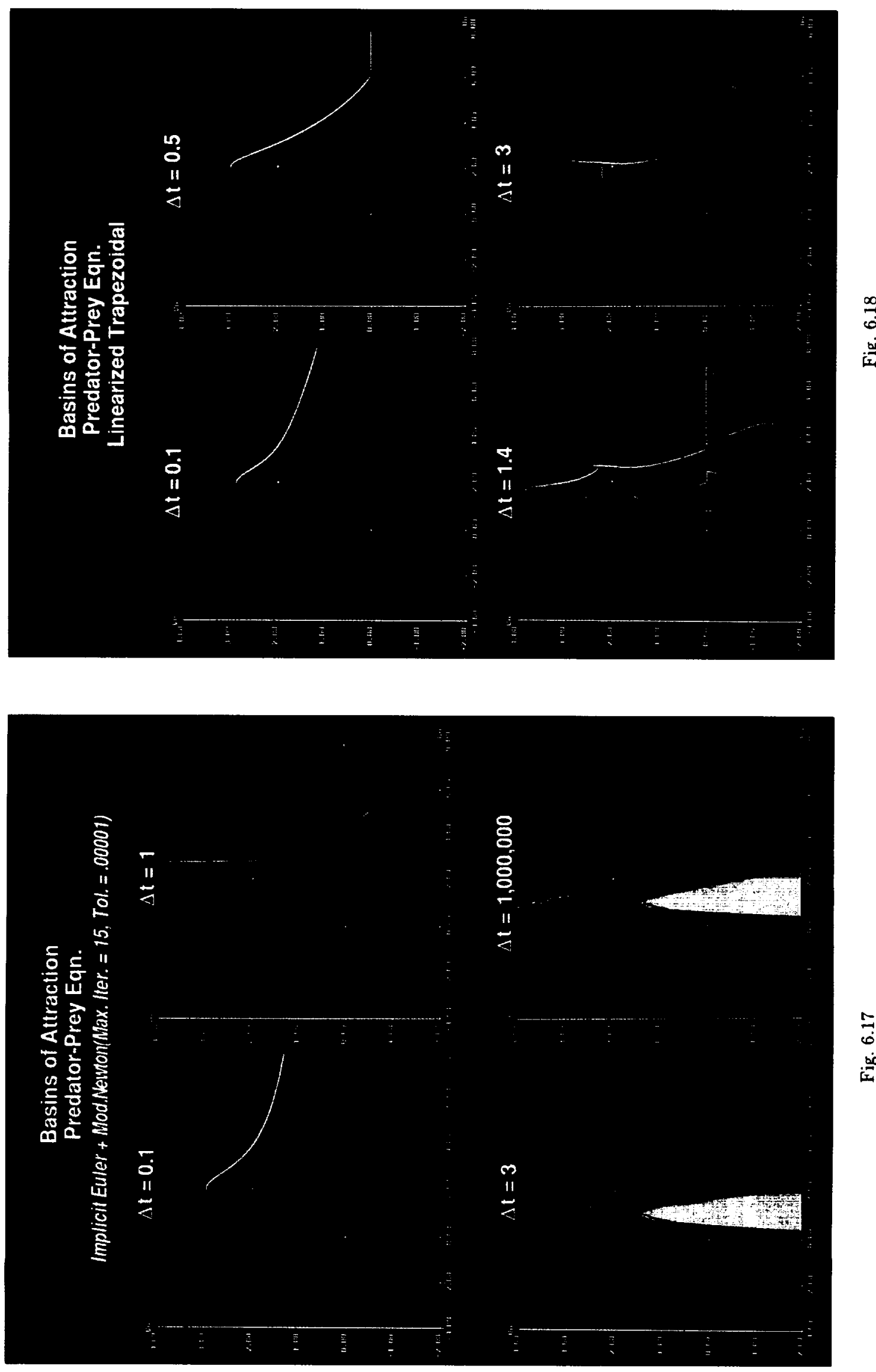


$$
\begin{array}{cc}
\frac{5}{5} & \\
8 & 0 \\
11 & 11 \\
\frac{0}{5} & 7
\end{array}
$$

$\frac{5}{9} \div$

응 항

는

Е칯

40

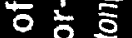

$0+6$

$5 \frac{1}{8}$

4

슨

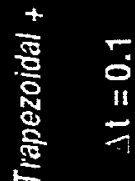

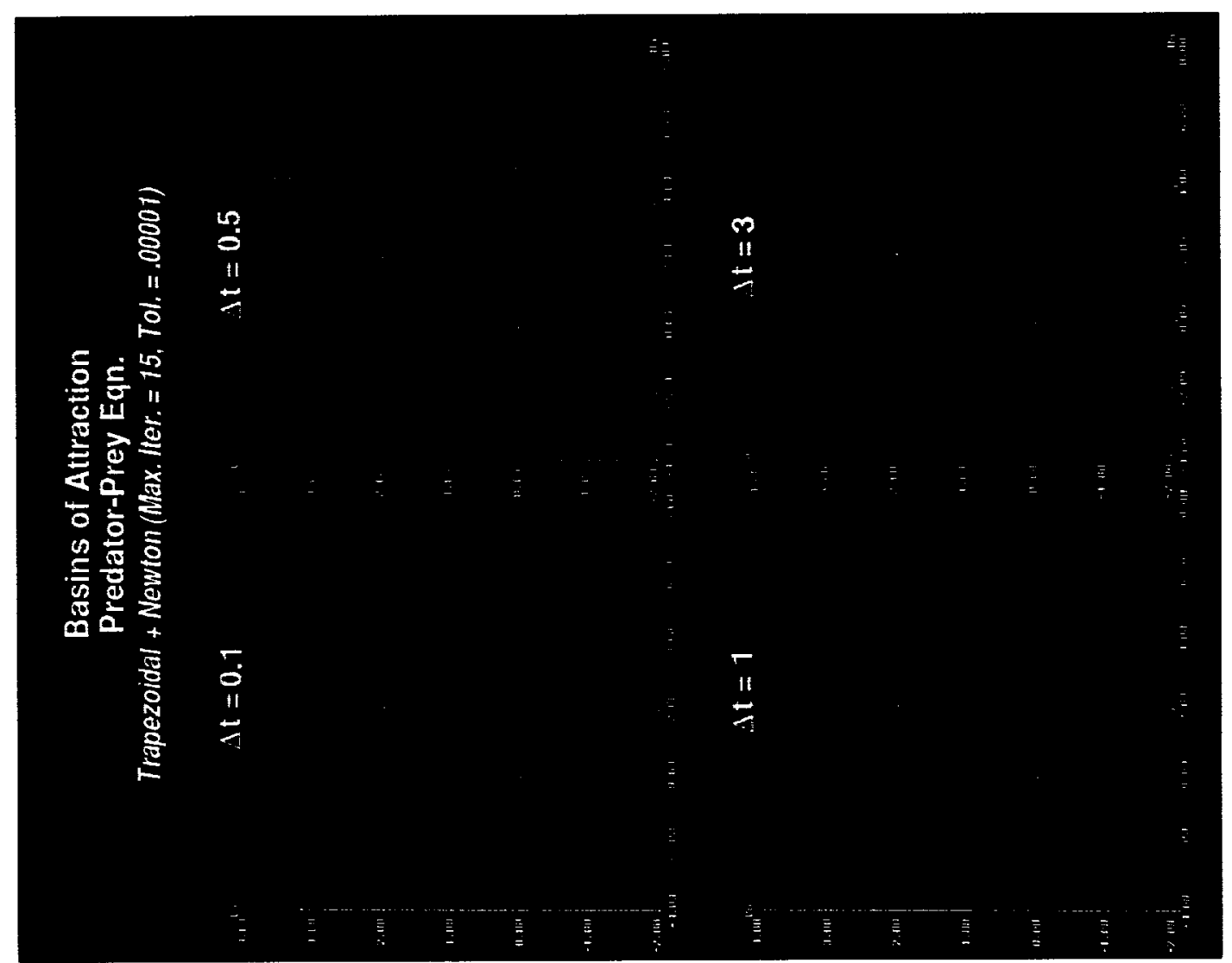



for larger $\Delta t$, the linearized implicit Euler, "implicit Euler + Newton", and "straight Newton" give the identical numerical basins of attraction. See also Yee \& Sweby [1992] for some discussion and illustration. Although the trapezoidal method, 3-level $\mathrm{BDF}$, and the midpoint implicit method did not turn the two saddle points $(1,0)$ and $(3,0)$ into stable fixed points of different type, they did turn the two saddle points into unstable fixed points of different types.

The evolution of the numerical basin of attraction (as $\Delta t$ changes) is very dramatic by each of the four LMMs. Take for example the trapezoidal method (Figs. 6.18-6.20), where the scheme becomes effectively unstable for large $\Delta t$. The size of the numerical basins of attraction for the stable fixed points $U_{E}$ shrink to almost nonexistence. This phenomenon might be one of the contributing factors to the unpopularity of the trapezoidal method in CFD. The basins are so fragmented and small for large $\Delta t$ that they are beyond the accuracy of the CM-2 to resolve and no further attempt was made. A better approach in computing these types of basins is to use interval arithmetic or the enclosure type method [Adams, 1990].

Our results for the predator-prey model indicate that linearized implicit methods are more robust and are more efficient than the other three iteration procedures and have a higher success rate of leading to physically correct steady states. In general, it seems that if one uses a $\Delta t$ far below what the linearized stability limit predicts, one has a better chance of avoiding spurious dynamics. Otherwise, the knowledge of numerical basins of attraction is vital in avoiding spurious dynamics when using a fixed time step that is larger than the stability limit of the standard explicit methods studied in Yee \& Sweby [1992]. Comparing the current results with those in Yee \& Sweby [1992], the implication is that unconditionally stable implicit methods are, in general, safer to use and have larger numerical basins of attraction than explicit methods. However, one cannot use too large a time step since the numerical basins of attraction can be so small that the initial data has to be very close to the exact solution for convergence.

Numerical experiments performed on the variable time step control (5.20) and (5.21) for model (4.2) also indicate that, although variable time step controls are not foolproof, they might alleviate the spurious dynamics most of the time. One shortcoming is that in order to avoid spurious dynamics, the required size of $\Delta t$ is impractical to use, especially for the explicit method (5.21).

\subsection{Numerical results for the perturbed Hamiltonian equation}

Selected results for the two representations of numerical basins of attraction for the perturbed Hamiltonian model (4.3) (viscous Burgers' equation with 3-point central in space) are shown in Figs. $6.23-6.33$ for $\varepsilon=0.1$. Figures $6.23-6.25 \mathrm{com}$ pare the noniterative and iterative solution procedures for solving the nonlinear algebraic equations using implicit Euler, trapezoidal, and 3-level BDF methods. Figures 6.26-6.33 show selected results of the numerical asymptotes with basins of attraction superimposed for four different $\Delta t$ 's. In all of Figs. 6.23-6.33, red regions represent the numerical basins of attraction for the stable spiral $(1 / 3,1 / 3)$. The numerical basins of attraction in Figs. 6.28, 6.30 , and 6.33 with $\Delta t=0.1$ appear to be the same as the exact basins of attraction. The numerical basin of attraction for $(1 / 3,1 / 3)$ is larger than the corresponding exact basin of attraction for $\Delta t=1$ by the implicit Euler and the 3-level BDF methods. See Figs. 6.26, 6.27, 6.31, and 6.32. Note also that the possibility of the numerical basin of attraction being larger than the exact one does not always occur when the time step is the smallest. For larger $\Delta t$ the linearized implicit Euler, "implicit Euler + Newton", and "straight Newton" give the same numerical basins of attraction. A conclusion similar to that arrived at in Sec. 6.2 for noniterative and iterative procedures applies here. Since the midpoint implicit method behaves similarly to the trapezoidal method and the 3-level BDF exhibits behavior similar to that discussed in the previous section, our discussion is restricted to the implicit Euler and trapezoidal methods.

\section{Implicit Euler Method:}

This is yet another interesting illustration of the use of an unconditionally stable implicit method where in practical computations, when the initial data are not known, the scheme has a higher chance of obtaining a physically correct solution if one uses a $\Delta t$ restriction slightly larger than that for the stability limit of an explicit method. Figures 6.23 and 6.26-6.28 show the two representations of numerical basins of attraction using the implicit Euler method. These figures show the generation of stable 


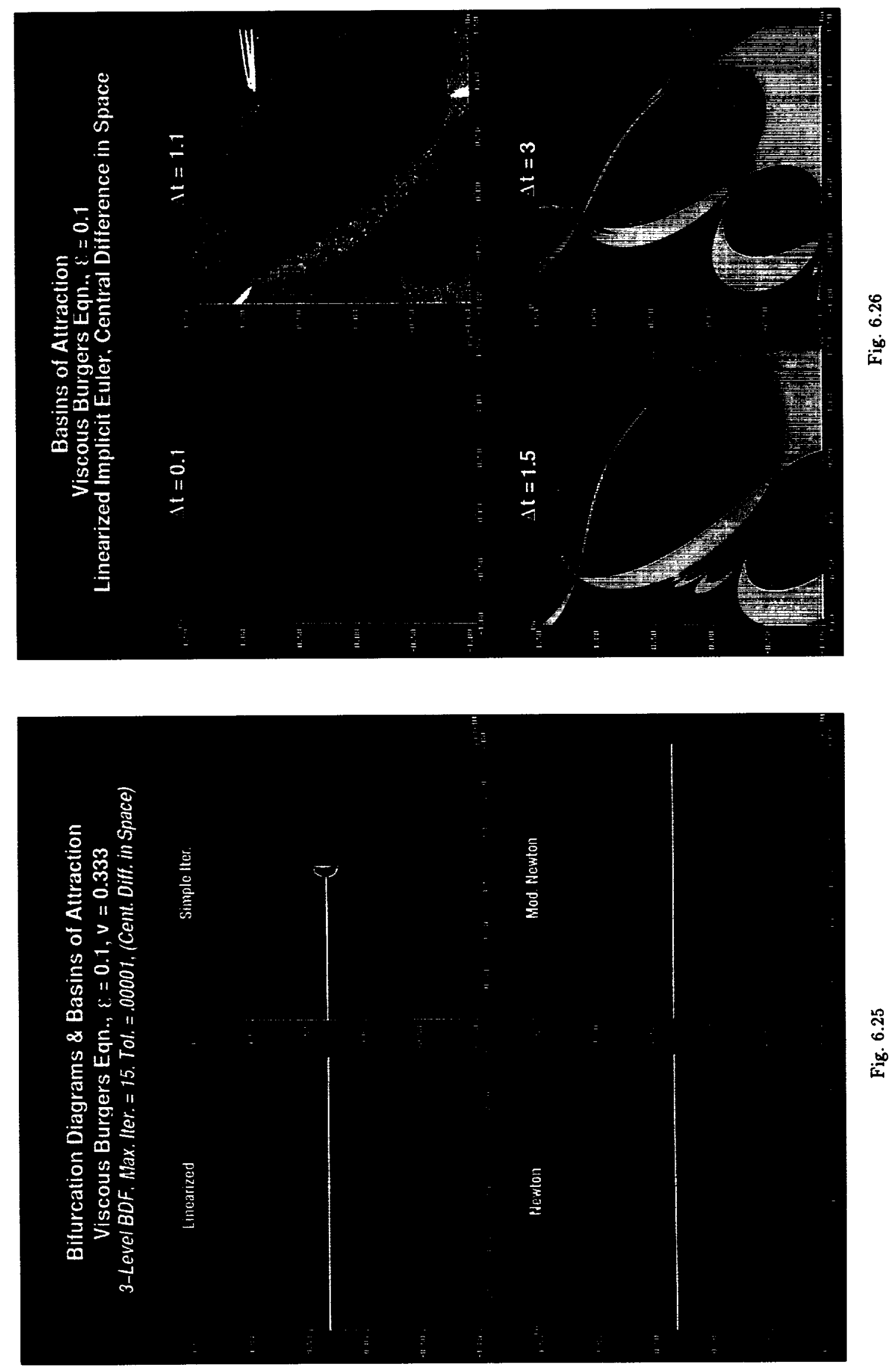

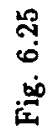




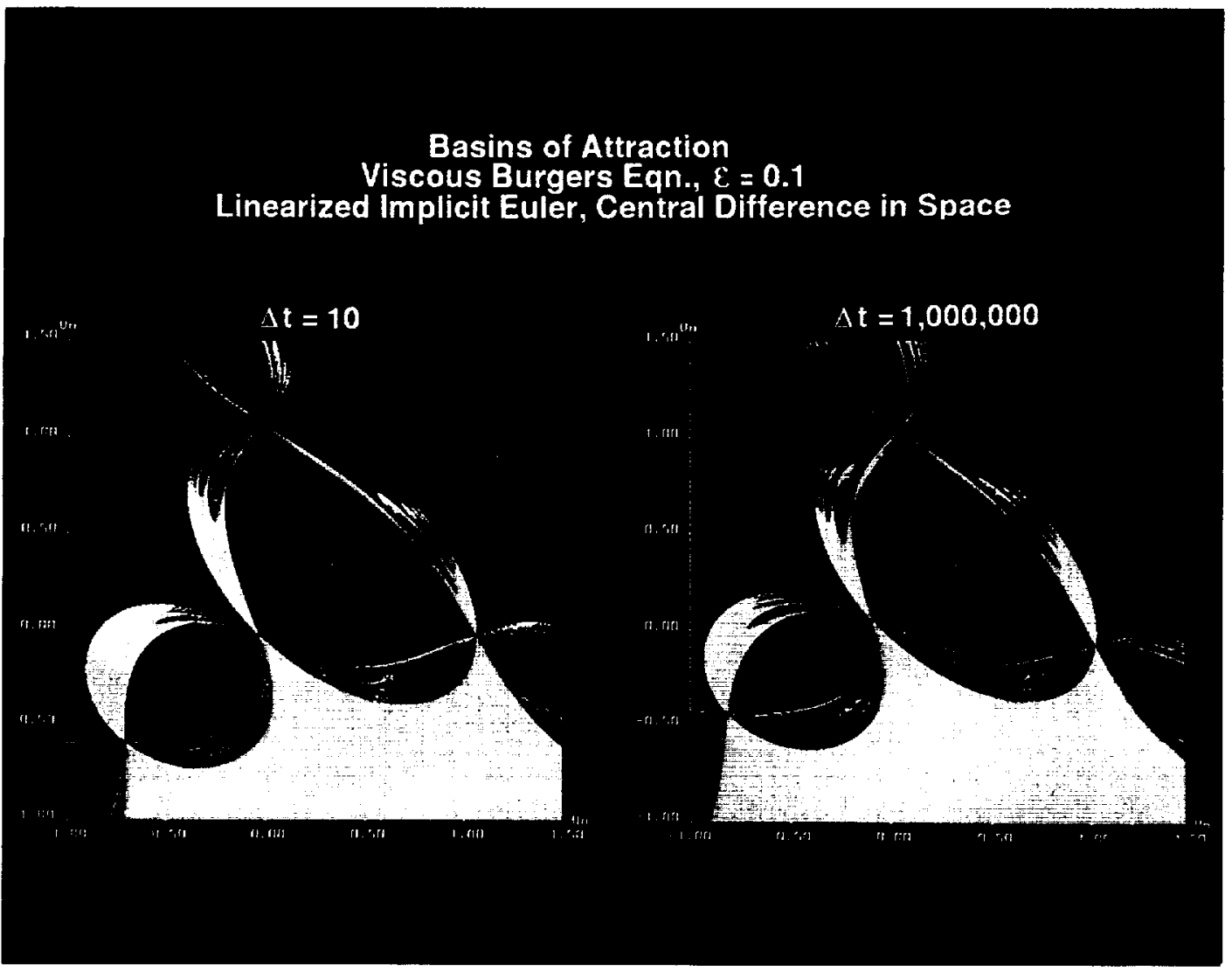

Fig. 6.26. (Continued)

spurious asymptotes for $\Delta t \geq 1$. As $\Delta t$ increases further, the size of the same numerical basin decreases and becomes fractal-like, and new numerical basins are generated. The numerical basin of attraction for $(1 / 3,1 / 3)$ was permanently altered for $\Delta t$ near or larger than 10 . In general, the linearized implicit Euler is more efficient and less likely to converge to a spurious asymptote than the other combinations of LMMs and solution procedures.

\section{Trapezoidal Method:}

Figures $6.24,6.29$, and 6.30 show the two representations of numerical basins of attraction using the trapezoidal method. In a manner similar to the linearized implicit Euler method, this scheme has a higher probability of obtaining a physically correct solution if one uses a $\Delta t$ similar to that of an explicit method. The numerical basins of attraction for $(1 / 3,1 / 3)$ computed by the linearized trapezoidal method are much larger than the corresponding exact basin of attraction for $\Delta t \leq 2$.
Their sizes are bigger than the ones generated by the implicit Euler method with the same $\Delta t$ values. The same behavior exists for the 3-level BDF method. As $\Delta t$ increases, the shrinkage of the numerical basins of attraction is more dramatic than for the other two LMMs. In most cases with larger $\Delta t$, the allowable initial data required to avoid spurious dynamics is impractical to use since the "safe" initial data has to be very close to the exact steady-state solution. Again, this phenomenon might be one of the contributing factor to the unpopularity of the trapezoidal method in CFD applications. For large $\Delta t$, the linearized trapezoidal scheme becomes effectively unstable due to the fragmentation of the numerical basins of attraction (see Yee \& Sweby [1992] for the plots). Again, due to the high cost of double precision computations, no further attempts were made for large $\Delta t$. The computation of these basins requires an interval arithmetic or the enclosure [Adams, 1990] type of mathematical operation before a more precise behavior can be revealed. 

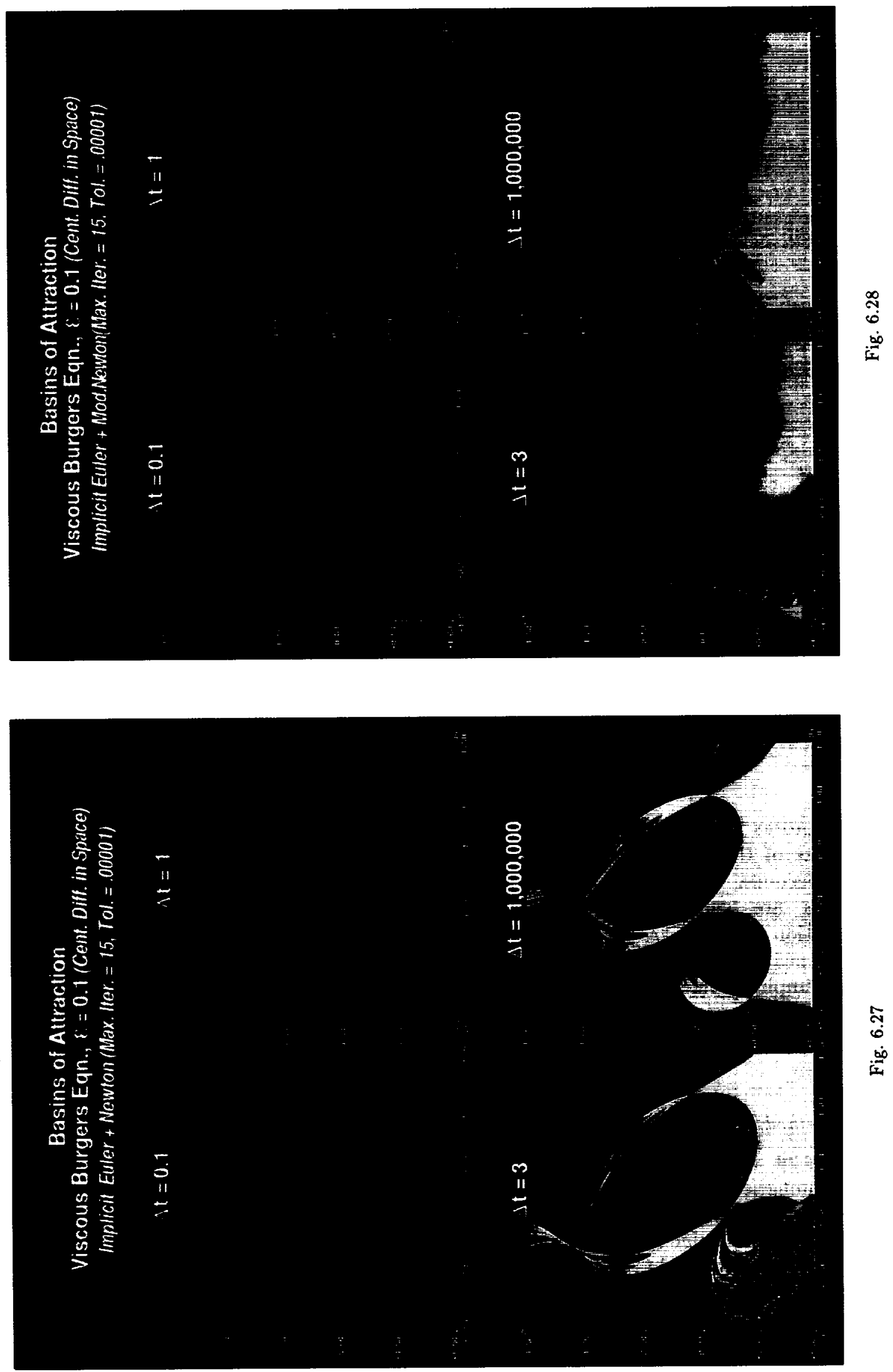
W"

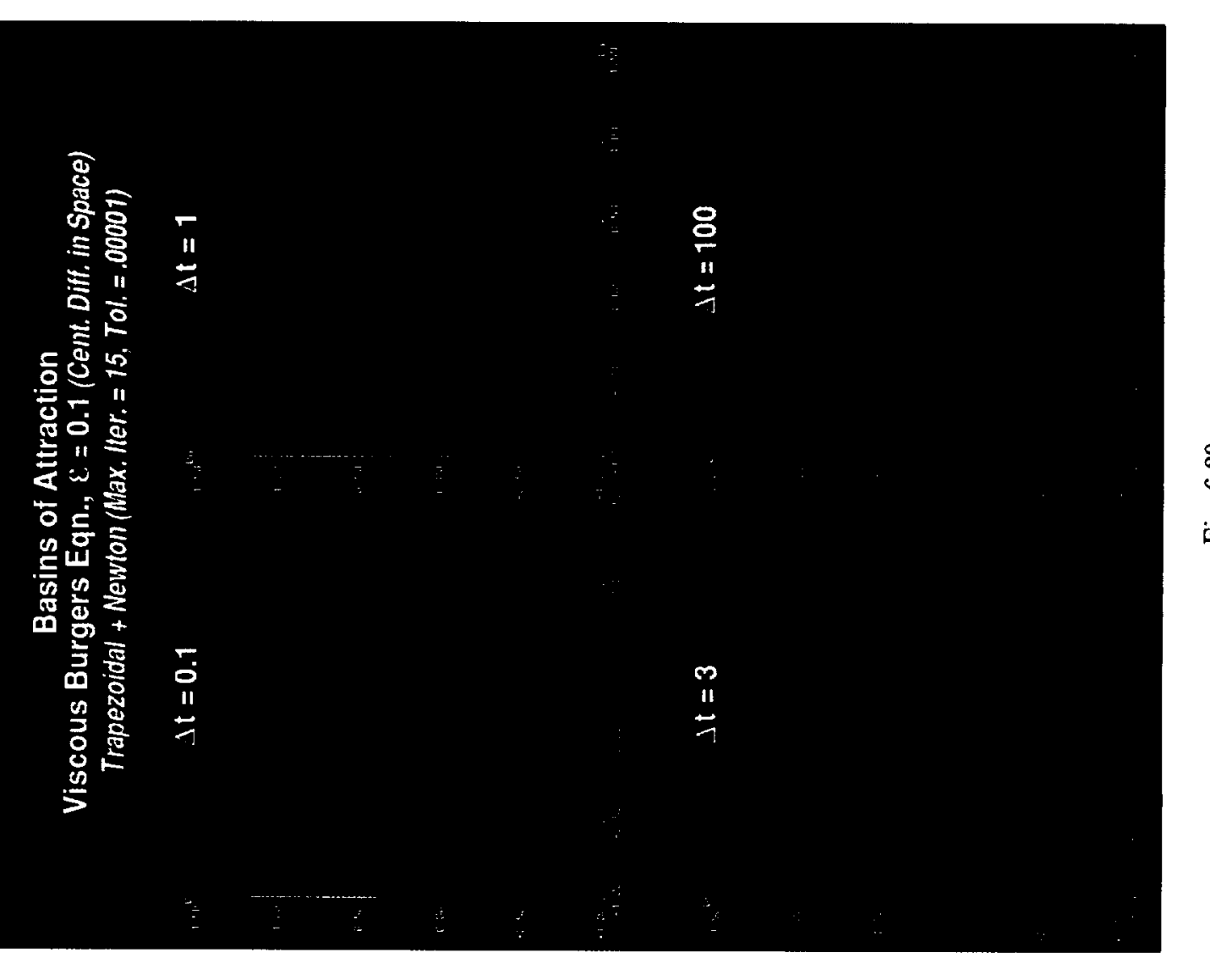



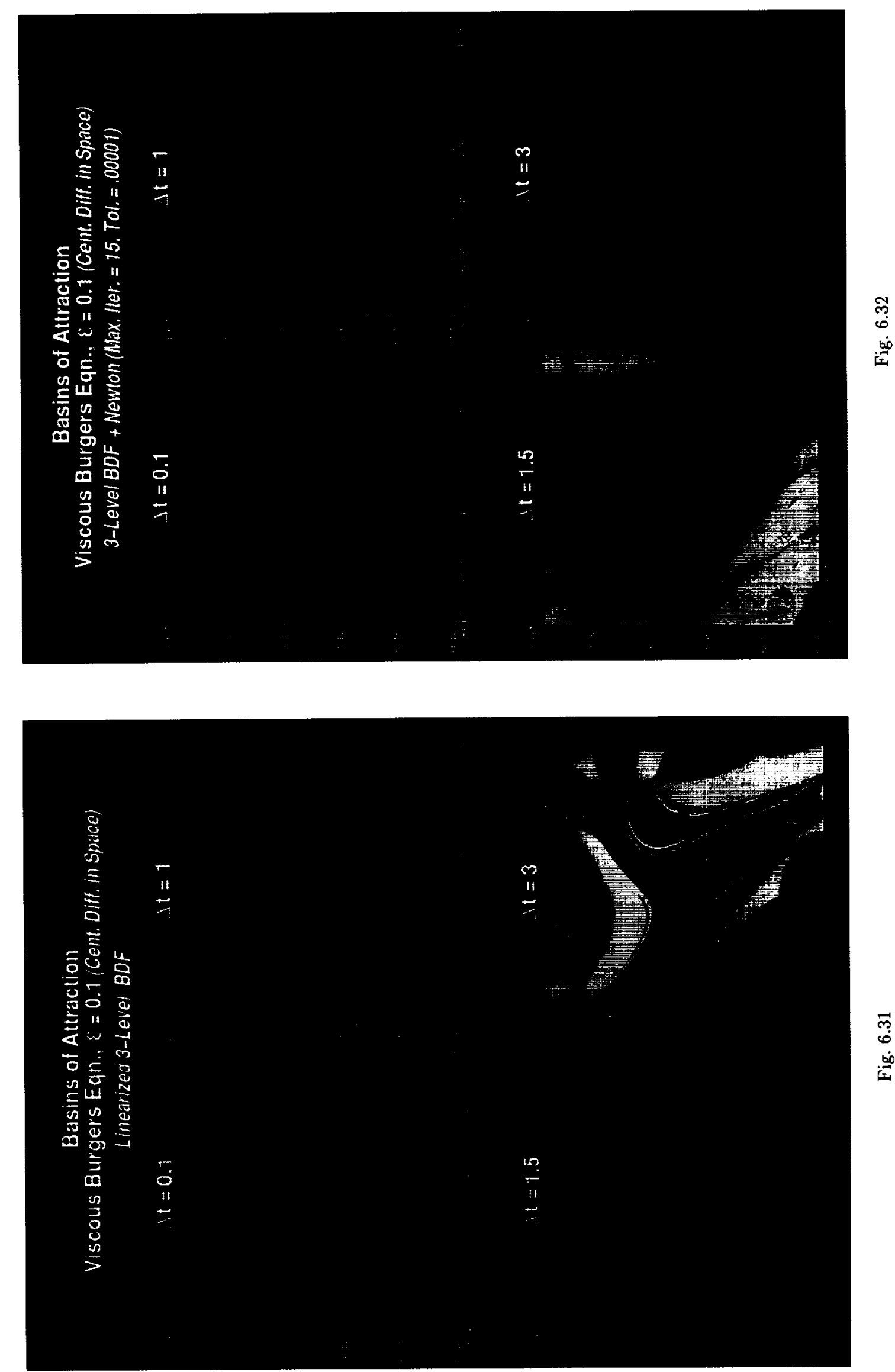


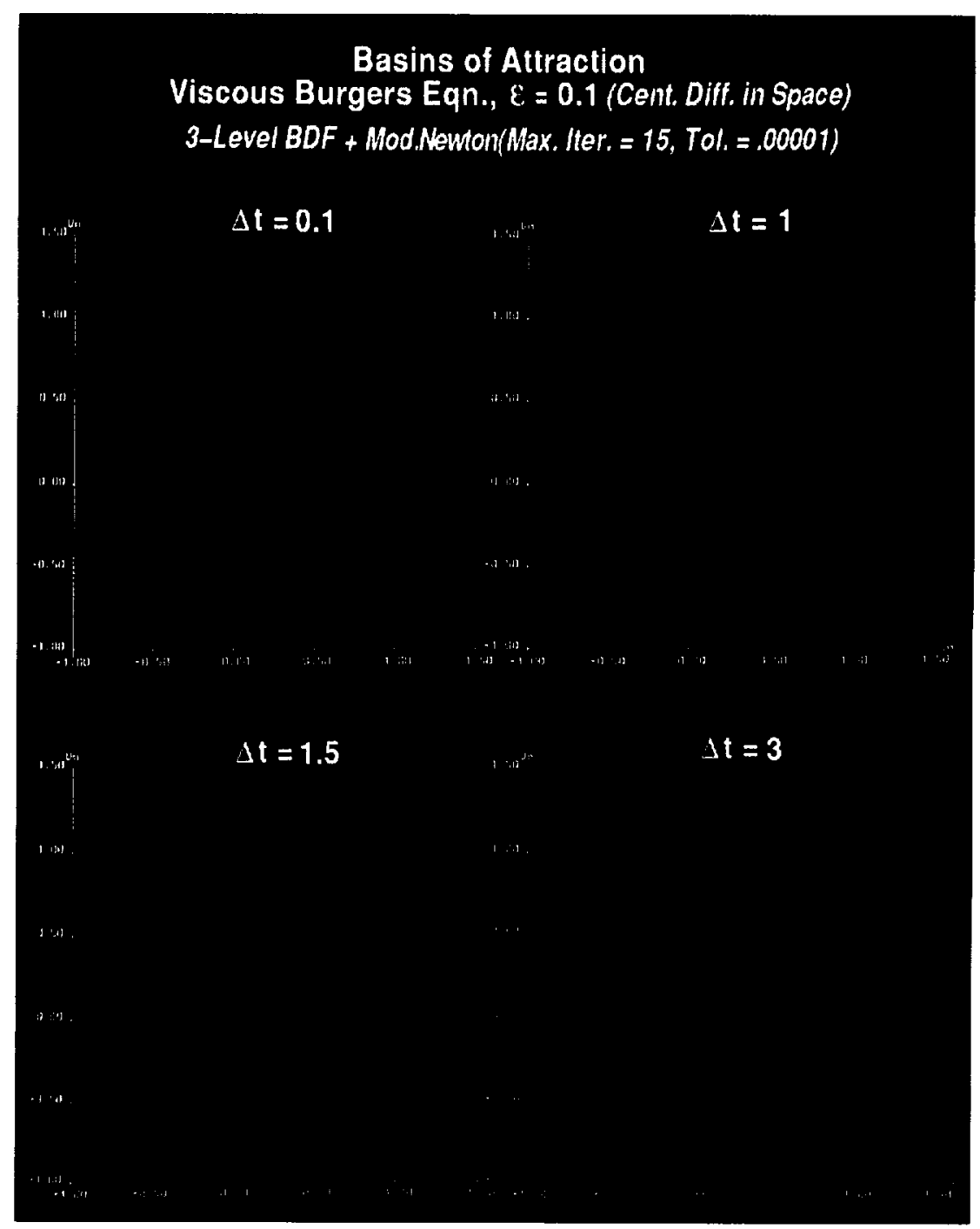

Fig. 6.33

\section{General Discussion of Numerical Results}

Studies showed that all of the four implicit LMMs exhibit a drastic distortion but less shrinkage of the basin of attraction of the true solution than standard explicit methods studied in Yee \& Sweby [1992]. In some cases with smaller $\Delta t$ (near the linearized stability limit of standard explicit methods), the implicit LMMs exhibit enlargement of the basins of attraction of the true solution. Overall, the numerical basins of attraction of a noniterative implicit procedure mimics more closely the basins of attraction of the continuum than the studied iterative implicit procedures for the four implicit LMMs. In general, the numerical basins of attraction bear no resemblance to the exact basins of attraction.
The size can increase or decrease depending on the time step. Also, the possible existence of the largest numerical basin of attraction that is larger than the exact one does not occur when the time step is the smallest. The dynamics of numerics of the implicit methods differ significantly from each other, and the different methods of solving the resulting nonlinear algebraic equations are very different from each other since different numerical methods and solution procedures result in entirely different nonlinear discrete maps. Although unconditionally stable implicit methods allow theoretically large $\Delta t$, the numerical basins of attraction (allowable initial data) for large $\Delta t$ sometimes are so fragmented and/or so small that the safe (or practical) choice of $\Delta t$ is slightly larger or comparable to the stability limit 
of standard explicit methods. In general, if one uses a $\Delta t$ that is a fraction of the stability limit, one has a higher chance of convergence to the correct asymptote. Studies in Yee \& Sweby [1992] for standard explicit methods confirmed this phenomenon.

One of the causes of the above behavior of implicit LMMs is the existence of stable and unstable spurious asymptotes other than steady states which have a similar detrimental (in terms of robustness) effect as explicit methods. Another cause of the observed behavior is due to the fact that an unstable fixed point can become a stable fixed point and can change type, e.g., from a saddle to a stable or unstable node. One consequence of the stabilization of unstable fixed points is a distortion, shrinkage and/or segmentation of the resulting numerical basin of attraction of the true steady states. Another consequence of this behavior is that the flow pattern can change topology as the discretized parameter is varied. Thus, even though LMMs preserve the same number (but not the same types) of fixed points as the underlying DEs, the numerical basins of attraction of LMMs do not coincide with the exact basins of attraction of the DEs even for small $\Delta t$. Some of the dynamics of the LMMs observed in our study can be used to explain the root of why one cannot achieve the theoretical linearized stability limit of the typical implicit LMMs in practice when solving strongly nonlinear DEs (e.g., in CFD).

Additional general remarks can be made for the following comparisons, independent of the four implicit LMMs:

\section{Simple Iteration versus Other Studied Methods:}

The stability and numerical basins of attraction by simple iteration are similar to those of standard Runge-Kutta and other commonly used explicit methods. The only advantage in using the "implicit method + simple iteration" over nonLMM explicit methods is that spurious steady states cannot occur.

\section{Noniterative versus Iterative:}

If one uses an implicit LMM for the time-dependent approach to obtaining steady-state numerical solutions, the linearized (or noniterative) version of the implicit methods are more efficient and less likely to converge to a spurious asymptote or get trapped in a spurious limit cycle than the other three studied iterative procedures. Overall, the noniterative implicit Euler scheme is more stable and less likely to converge to a spurious asymptote than the other combinations of LMMs and solution procedures. The phenomenon can explain more precisely a contributing factor to the popularity of the noniterative implicit Euler in CFD applications.

\section{Straight Newton versus Other Studied Methods:}

Studies indicated that contrary to popular belief, the initial data using the straight Newton method may not have to be close to the exact solution for convergence. Straight Newton also exhibits stable and unstable spurious asymptotes. Initial data can be reasonably removed from the asymptotic values and still be in the basin of attraction. However, the basins can be fragmented even though the corresponding exact basins of attraction are single closed domains. The cause of nonconvergence may just as readily be due to the fact that the numerical basins of attraction are fragmented. In many cases, the results obtained are better than those obtained by the trapezoidal and 3-level BDF methods (regardless of the three iterative procedures). If one uses a time step slightly bigger than the stability limit of standard explicit methods for the four LMMs, straight Newton can have similar or better performance. In fact, using a large $\Delta t$ by the linearized implicit Euler method or the implicit Euler + Newton procedure has the same chance of obtaining the correct steady state as the straight Newton method if the initial data are not known or arbitrary initial data is taken.

\section{Variable Time Step Control:}

Our studies showed that the variable time step control method (5.20) can occasionally stabilize unstable fixed points, depending on the initial data, starting time step, and the value of "tol 1 ". One shortcoming is that the size of $\Delta t$ needed to avoid spurious dynamics is impractical to use, especially for the explicit method (5.21).

\section{Acknowledgments}

The authors wish to thank Arieh Iserles and Andrew Stuart for their valuable discussions during the course of this research. Special thanks to André Lafon and Marcel Vinokur for their critical review of the manuscript. Financial support from Thomas Lasinski for the second author as a visiting scientist at RIACS/NASA Ames is greatfully acknowledged. 


\section{References}

Adams, E. [1990] "Periodic solutions: Enclosure, verification, and applications," in Computer Arithmetic and Self-Validating Numerical Methods (Academic Press) pp. 199-245.

Budd, C. J., Stuart, A. M., Koomullil, G. P. \& Yee, H. C. [1994] "Numerical solution behaviour of model convection-diffusion BVP with grid adaptation," in preparation.

Dieci, L. \& Estep, D. [1990] "Some stability aspects of schemes for the adaptive integration of stiff initial value problems," School of Math. Report (Georgia Institute of Technology).

Flashner, H. \& Guttalu, R. S. [1988] "A computational approach for studying domains of attraction for nonlinear systems," Int. J. Non-Linear Mechanics 23(4), 279-295.

Griffiths, D. F., Sweby, P. K. \& Yee, H. C. [1992a] "On spurious asymptotic numerical solutions of explicit Runge-Kutta methods," IMA J. in Numerical Analysis 12, 319-338.

Griffiths, D. F., Stuart, A. M. \& Yee, H. C. [1992b] "Numerical wave propagation in hyperbolic problems with nonlinear source terms," SIAM J. of Numerical Analysis.

Guckenheimer, J. \& Holmes, P. [1983] Nonlinear oscillations, dynamical systems, and bifurcations of vector fields (Springer-Verlag, New York).

Hale, J. \& Kocak, H. [1991] Dynamics and Bifurcations (Springer-Verlag, New York).

Hsu, C. S., Guttalu, R. S. \& Zhu, W. H. [1982] "A method of analyzing generalized cell mappings," J. App. Mech. 49, 885-894.

Iserles, A. [1988] "Stability and dynamics of numerical methods for nonlinear ordinary differential equations," DAMTP NA1, 1988 (University of Cambridge, Cambridge England).

Iserles, A., Peplow, A. T. \& Stuart, A. M. [1990] "A unified approach to spurious solutions introduced by time discretisation, Part I: Basic theory," DAMTP 1990/NA4, Numerical Analysis Reports (University of Cambridge, Cambridge England).

Keller, H. B. [1977] "Numerical solution of bifurcation and nonlinear eigenvalue problems," in Applications of Bifurcation Theory, ed. Rabinowitz, P. H., (Academic Press) pp. 359-384.

Lafon, A. \& Yee, H. C. [1991] "Dynamical approach study of spurious steady-state numerical solutions for nonlinear differential equations, Part III: The effects of nonlinear source terms and boundary conditions in reaction-convection equations," NASA TM-103877 July 1991; to appear in Int. J. CFD.

Lafon, A. \& Yee, H. C. [1992] "Dynamical approach study of spurious steady-state numerical solutions of nonlinear differential equations, Part IV: Stability versus numerical treatment of nonlinear source terms," ONERA-CERT Technical Report DERAT 45/5005.38 Feb. 1992; to appear in Int. J. CFD.

Mitchell, A. R. \& Griffiths, D. F. [1985] "Beyond the linearized stability limit in nonlinear problems," Report NA/88 July 1985 (Department of Mathematical Sciences, University of Dundee, Scotland U.K.)

Stuart, A. M. [1989] "Linear instability implies spurious periodic solutions," IMA J. Num. Anal. 9, 465-486.

Sweby, P. K. \& Yee, H. C. [1991] "On spurious asymptotic numerical solutions of $2 \times 2$ systems of ODEs," Numerical Analysis Report 7/91, October 1991 (University of Reading, England).

Sweby, P. K. \& Yee, H. C. [1994] "On the dynamics of some grid adaptation schemes," Proceedings of the 4 th International Conference on Numerical Grid Generation in CFD and Related Fields, University College of Swansea, UK, April 6-8, 1994.

Yee, H. C., Sweby, P. K. \& Griffiths, D. F. [1991] "Dynamical approach study of spurious steady-state numerical solutions for nonlinear differential equations, Part I: The dynamics of time discretizations and its implications for algorithm development in computational fluid dynamics," NASA TM-102820, April 1990; also J. Comput. Phys. 97, 249-310.

Yee, H. C., Sweby, P. K. \& Lafon, A. [1992] "Basins of attraction and the time-dependent approach to obtaining steady-state numerical solutions," Proceedings of the ICFD Conference on Numerical Methods for Fluid Dynamics, April 7-10, 1992, Reading, England.

Yee, H. C. \& Sweby, P. K. [1992] "Dynamical approach study of spurious steady-state numerical solutions for nonlinear differential equations, Part II, global asymptotic behavior of time discretizations," RNR Technical Report RNR-92-008, March 1992; to appear in Int. J. $C F D$.

Yee, H. C. \& Sweby, P. K. [1993] "On the dynamics of some iterative implicit schemes," Proceedings of the Chaotic Numerics Workshop July 12-16, 1993 (Deakin University, Geelong, Australia), AMS special publications COMM 172, 1994. 
